\title{
On the Spectrum of the Plenoptic Function
}

\author{
Christopher Gilliam*, Member, IEEE, Pier-Luigi Dragotti, Senior Member, IEEE, and \\ Mike Brookes, Member, IEEE
}

\begin{abstract}
The plenoptic function is a powerful tool to analyze the properties of multi-view image datasets. In particular, the understanding of the spectral properties of the plenoptic function is essential in many computer vision applications including image-based rendering. In this paper, we derive for the first time an exact closed-form expression of the plenoptic spectrum of a slanted plane with finite width and use this expression as the elementary building block to derive the plenoptic spectrum of more sophisticated scenes. This is achieved by approximating the geometry of the scene with a set of slanted planes and evaluating the closed-form expression for each plane in the set. We then use this closed-form expression to revisit uniform plenoptic sampling. In this context, we derive a new Nyquist rate for the plenoptic sampling of a slanted plane and a new reconstruction filter. Through numerical simulations, on both real and synthetic scenes, we show that the new filter outperforms alternative existing filters.
\end{abstract}

Index Terms-Spectral Analysis, Plenoptic Function, ImageBased Rendering, Plenoptic Sampling.

\section{INTRODUCTION}

$\mathbf{I}$ MAGE-Based Rendering (IBR) is an effective technique for rendering novel views of a scene from a set of multiview images. The novel views are rendered by interpolating nearby images. The advantage of such a method is that, since it combines real images, it can produce convincing photorealistic results without requiring a detailed 3D model of the scene. For this reason, IBR finds application in immersive communication systems [1], 3DTV [2] and free viewpoint TV (FTV) [3]. For surveys on the topic we refer the reader to [4], [5]. A drawback of IBR, however, is that a very large number of images are needed to compensate for the lack of geometric information [4]. Therefore, an important goal is to reduce the required number of multi-view images whilst still achieving good quality rendering.

The principle underlying IBR is that a scene can be represented as a collection of light rays emanating from its surface. The multi-view image set, therefore, samples this representation of the scene with each image recording the intensity of a set of light rays travelling from the scene to the camera [4]. The light rays in question may be parametrized

Copyright (c) 2013 IEEE. Personal use of this material is permitted. However, permission to use this material for any other purposes must be obtained from the IEEE by sending a request to pubs-permissions@ieee.org

Manuscript received February 12, 2013; revised September 03, 2013; accepted November 11, 2013.

C. Gilliam is with the Department of Electronic Engineering, The Chinese University of Hong Kong, Shatin, New Territories, Hong Kong (email: cgilliam@ee.cuhk.edu.hk). The work was carried out whilst at the Department of Electrical and Electronic Engineering, Imperial College London, London, SW7 2AZ UK.

P. L. Dragotti and M. Brookes are with the Department of Electrical and Electronic Engineering, Imperial College London, London, SW7 2AZ UK (email: p.dragotti@imperial.ac.uk; mike.brookes@imperial.ac.uk).

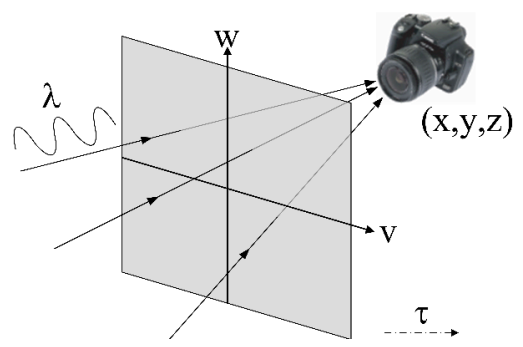

Fig. 1. Diagram showing the 7D plenoptic function, where $(x, y, z)$ is the viewing position and $(v, w)$ is the viewing direction in terms of pixel coordinates. The final two parameters define the wavelength, $\lambda$, and the time, $\tau$.

using the 7D plenoptic function [6], illustrated in Fig. 1. This describes the intensity of a light ray passing through the camera center at a 3D spatial location, $(x, y, z)$, in a certain $2 \mathrm{D}$ viewing direction, $(v, w)$, with a certain wavelength, $\lambda$, and at a certain time, $\tau$. IBR can thus be regarded as a sampling and interpolation problem: a finite set of images, with finite resolution, sample the continuous plenoptic function and the rendering of a new viewpoint entails the reconstruction of the function from the samples [4]. If the function is incorrectly sampled then blurring or ghosting artefacts appear in the rendered views [7]. Accordingly, by studying the sampling of the plenoptic function, termed plenoptic sampling, we can determine the minimum number of images required for artefact-free rendering.

The first spectral analysis of plenoptic function was performed by Chai et al. [7]. Assuming uniform camera placement, they analyzed the spectral support of the plenoptic function to find the Nyquist sampling rate for a Lambertian surface $^{1}$ with no occlusions. This spectral analysis was extended to non-Lambertian scenes in [8], [9] and scenes with occlusions in [8]. In particular, the authors of [9] showed that, unless the scene surface was flat, the continuous plenoptic spectrum was band-unlimited. More recently the focus of plenoptic sampling has been on the acquisition set-up. For instance, cameras with arbitrary position and orientation are considered in [10], and Bagnato et al. [11] examined plenoptic sampling when the cameras are placed on a sphere (as opposed to a plane in [7], [8], [9], [10]). A detailed review of plenoptic sampling, including non-uniform camera placement, was presented by Zhang and Chen in [12].

In this paper, we examine the spectral properties of the plenoptic function for a specific scene: a 1D planar facet, which we term a slanted plane. To perform this spectral analysis, we use the framework presented in [9] combined with

\footnotetext{
${ }^{1}$ A Lambertian surface has the property that the intensity of a light ray leaving a point on the surface is independent of the viewing angle.
} 
two realistic constraints: finite scene width (FSW) and finite camera field-of-view (FFV). The novelty of our approach is that the constraints are incorporated directly when performing the spectral analysis. Under this new set-up, we are able to obtain an exact closed-form expression for the plenoptic spectrum of a slanted plane. Although this scene may seem simple, our interest comes from its ability to be used as an elementary element from which to derive the plenoptic spectrum of more complicated geometries. In particular, we show that the plenoptic spectrum for a smoothly varying scene can be obtained by first approximating its geometry using a sequence of slanted planes and then evaluating our closed-form expression for each plane in the sequence.

We also examine the essential bandwidth of the plenoptic spectrum of a slanted plane and derive sampling results. In particular, we obtain a new reconstruction filter for uniform plenoptic sampling and a new expression for the Nyquist sampling rate. Using synthetic and real scenes, we demonstrate the improvement of this new filter over existing ones when reconstructing the plenoptic function. A preliminary study of the plenoptic spectrum of a slanted plane was presented in [13]. Our paper builds upon this preliminary work to provide a thorough analysis of the spectrum, in particular its spectral support and how this can be used in sampling.

In the context of IBR, it is worth noting that algorithms presented in [14], [15], [16], [17] partition the scene into a set of layers parallel to the cameras. This type of partition is equivalent to a piecewise constant approximation of scene depth. Such an approximation neglects the effect of depth variation on the plenoptic spectrum, which was recently highlighted in [9]. In view of this, our plenoptic results for slanted planes form a theoretical basis from which to incorporate planar scene models in practical IBR algorithms.

The paper is organized as follows: in the next section we outline the parametrization of the plenoptic function that will be used throughout this paper. Next, in Section III, we cover the current state-of-the-art in uniform plenoptic sampling, in particular the framework presented in [9]. We examine the spectrum of the plenoptic function under FSW and FFV in Section IV and derive its exact expression for a slanted plane. In Section V, we determine the essential bandwidth for this plenoptic spectrum and use it to determine the minimum sampling density for a slanted plane. These results are evaluated in Section VI using both synthetic and real scenes. Lastly, conclusions are given in Section VII.

\section{Parametrization of the Plenoptic Function}

The high dimensionality of the plenoptic function makes its theoretical analysis a challenging problem. A common simplification is to reduce the dimensionality by restricting certain aspects of the scene and sensing set-up [4]. With these restrictions, the 7D plenoptic function can be re-parametrized into more tractable representations, such as McMillan and Bishop's 5D plenoptic model [18] and the 3D concentric mosaic [19]. In this paper, we focus on two popular 4D representations: the light field [20] (similar to the lumigraph [21] or ray-space [22] representations) and the surface light

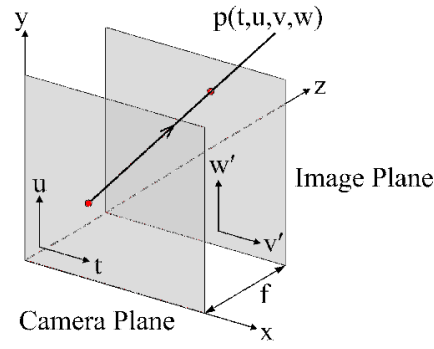

(a)

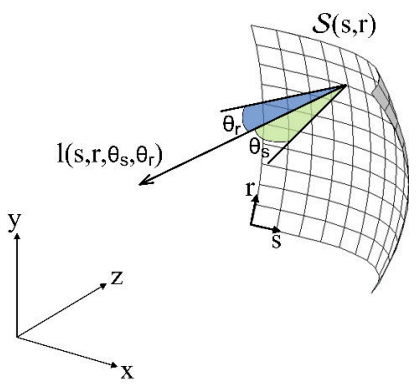

(b)
Fig. 2. Diagram (a) illustrates the 4D light field, $p(t, u, v, w)$, in which a light ray is defined by its location on the camera plane, $(t, u)$, and its pixel location on the image plane, $(v, w)=\left(v^{\prime}-t, w^{\prime}-u\right)$. The distance between the two planes is the focal length $f$. Diagram (b) illustrates the surface plenoptic function, $l\left(s, r, \theta_{s}, \theta_{r}\right)$, which is the intensity of a light ray emitted from a point $(s, r)$ on the scene surface $\mathcal{S}$ at a viewing direction $\left(\theta_{s}, \theta_{r}\right)$.

field [23], [24]. Both parametrizations assume that the scene is static, that the radiance of a light ray is constant along its path and that the image is monochromatic ${ }^{2}$. They differ, however, in the way in which they characterize a light ray.

Assuming a pin-hole camera model, the light field parametrization defines each light ray by its intersection with two parallel planes, the camera plane, $(t, u)$, and the image plane, $(v, w)$, whose separation is equal to the focal length, $f$. As a result the light field, $p(t, u, v, w)$, defines the intensity of the light ray at a camera location $(t, u)$ and pixel location $(v, w)$ [9]. An illustration of the light field is shown in Fig. 2(a). Notice that in this framework the light ray is defined with respect to the receiving camera position. In contrast, the light ray in the surface light field is defined relative to its point of origin on the scene surface, $\mathcal{S}$. This surface is parametrized using two curvilinear surface coordinates, $(s, r)$, therefore a point on the surface is defined as $\mathcal{S}(s, r)=[x(s, r), y(s, r), z(s, r)]^{T}$, where $[x, y, z]^{T}$ are the point's Cartesian coordinates [25]. The direction the light ray leaves the surface is defined by the viewing angle $\left(\theta_{s}, \theta_{r}\right)$, where $\theta_{s}$ and $\theta_{r}$ are measured relative to the $z$-axis. Therefore $l\left(s, r, \theta_{s}, \theta_{r}\right)$ is the intensity of the light ray emitted from a point $(s, r)$ on the scene surface at a viewing direction $\left(\theta_{s}, \theta_{r}\right)$. Fig. 2(b) shows a diagram of the surface light field.

A further simplification, used in [8], [7], [9], is to consider only a horizontal slice of the scene. In the case of the light field, $u$ and $w$ are fixed; this corresponds to the situation where the camera positions are constrained to a line parallel to the $x$-axis and only one scan-line is considered in each image. The resulting 2D light field, $p(t, v)$, is the intensity of the light ray at camera location $t$ and pixel location $v$. For the surface light field, $r$ and $\theta_{r}$ are fixed and a one dimensional surface, $\mathcal{S}(s)$, is assumed. Therefore the 2D surface light field, $l\left(s, \theta_{s}\right)$, is the intensity of the light ray emitted from a surface point $[x(s), z(s)]^{T}$, at a viewing direction $\theta_{s}$ [9]. Diagrams of the 2D light field and 2D surface light field are shown in Fig. 3. For the remainder of the paper we shall use the 2D parametrizations when analysing the light field and surface light field. Accordingly, in the 2D surface light field, we drop

\footnotetext{
${ }^{2}$ Alternatively, the R, G, and B channels can be treated independently.
} 


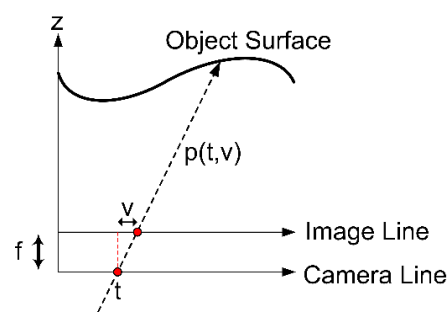

(a)

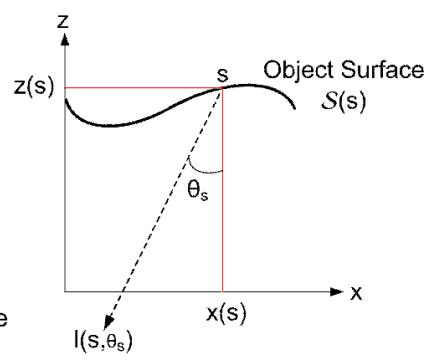

(b)
Fig. 3. Diagram (a) illustrates the 2D light field, $p(t, v)$, in which a light ray is defined by its intersection with the camera line at a location $t$ and the corresponding pixel location, $v$, on the image line. Diagram (b) illustrates the 2D surface plenoptic function, $l\left(s, \theta_{s}\right)$, in which a light ray is defined by its point of origin, $s$, on the object surface, $\mathcal{S}$, at a viewing angle $\theta_{s}$.

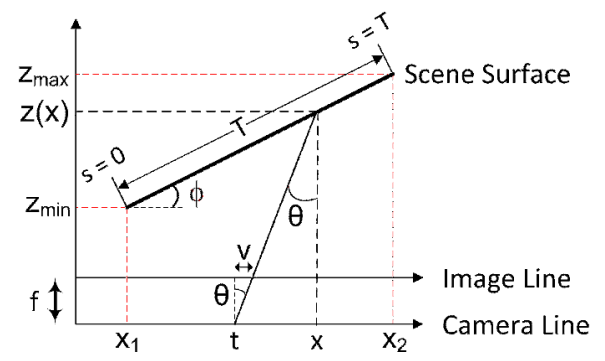

Fig. 4. Diagram of a slanted plane showing the intersection of a light ray $(t, v)$ with the scene surface at $(x, z(x))$, where $z(x) \in\left[z_{\min }, z_{\max }\right]$ is the depth of the surface. Note that $f$ is the focal length of the cameras, $\theta$ is the viewing angle and $\phi$ is the angle of slant.

the $s$ subscript from the viewing angle, referring to it only as $\theta$.

A visual representation of the $2 \mathrm{D}$ light field can be constructed if we plot its intensity at all possible $(t, v)$ coordinates. This visual representation is known as the Epipolar Plane Image (EPI) [26]. In the EPI, a point in the scene is mapped to a line with a gradient, $d v / d t$, that is inversely proportional to the depth of the point, $z$. This structure leads to the following important observation: lines with steeper gradients will always occlude lines with shallower gradients, in other words a point close to the camera will occlude any points that are more distant.

\section{UNIFORM PLENOPTIC SAMPLING IN A FOURIER FRAMEWORK}

When cameras are uniformly spaced, at a spacing $\Delta t$, it is natural to analyse plenoptic sampling within a classical Fourier framework. In such a framework, uniform sampling leads to spectral replication in frequency and the minimum sampling requirement, the Nyquist rate, is such that the replicas do not overlap. For this reason, many authors, such as [7], [8], [9], have studied the properties of the plenoptic spectrum, the Fourier transform of the plenoptic function. In particular, they have examined the spectral support of the plenoptic function since knowledge of this property can be used to determine the minimum number of cameras required to avoid aliasing.

\section{A. Spectral Analysis of the Plenoptic Function}

Chai et al [7] were the first to analyse the spectral support of the plenoptic function by using the inherent structure of the EPI. Assuming a Lambertian surface with no-occlusions, they showed that if the scene depth is limited by $z \in\left[z_{\min }, z_{\max }\right]$ then the plenoptic spectrum is approximately bounded by lines relating to the minimum and maximum depths, $z_{\min }$ and $z_{\max }$, respectively. More recently, in [8], [9], plenoptic spectral properties were revisited for a broader range of scenes, which included scenes with depth varying and/or non-Lambertian surfaces. Their analysis is based on exploiting the equivalence between the 2D light field and the 2D surface light field in order to derive properties of the plenoptic spectrum. This equivalence is formalised by modelling the scene with a functional surface. In this framework the depth of the scene surface, relative to the $x$ coordinate, is defined by the function $z(x)$ and its texture is modelled as a bandlimited signal, $g(s)$, where $s$ is the curvilinear coordinate on the surface. Now, assuming the camera line $t$ coincides with the $x$ coordinate system, the authors link a light ray arriving at $(t, v)$ to its point of origin on the surface at $(x, z(x))$ using the following geometric relationship

$$
t=x-z(x) \tan (\theta)=x-z(x) \frac{v}{f},
$$

where $\theta$ is the viewing angle. An illustration of this relationship is shown in Fig. 4. Provided this geometric relationship is a one-to-one mapping, the spatial position $(x, z(x))$ specifies a single curvilinear position $s$ on the surface, which allows the plenoptic function to be mapped to the surface light field and vice versa. The provision of a one-to-one mapping is enforced in [9] by excluding scenes with occlusions. Therefore $z(x)$ is constrained such that

$$
\left|z^{\prime}(x)\right|<\frac{f}{v_{m}},
$$

where $z^{\prime}(x)$ is the derivative of $z$ with respect to $x$, and $v_{m}$ is the maximum value of $v$ for a camera with a FFV, hence $v \in$ $\left[-v_{m}, v_{m}\right]$. Although this constraint is not directly enforced in [8], a one-to-one relationship is achieved by selecting the closest point to the scene that satisfies (1).

Equation (1) allows the mapping of the plenoptic function, $p(t, v)$, to $l_{x}(x, \bar{v})$ the intensity of a light ray emitted from the spatial position $(x, z(x))$ at a viewing direction defined by $\bar{v}=\tan (\theta)=v / f$, i.e.

$$
l_{x}(x, \bar{v})=p(x-z(x) \bar{v}, f \bar{v}) .
$$

The surface light field, $l(s, \theta)$, is then obtained by mapping the curvilinear coordinate, $s$, to the spatial position, $x$, and the viewing angle, $\theta$, to the viewing direction, $\bar{v}$, giving

$$
l(s, \theta)=l_{x}(x(s), \bar{v}(\theta)) .
$$

The importance of this mapping is that spectral properties of the plenoptic function can be derived by assuming properties of the surface light field without explicitly defining the scene's geometry.

For instance Do et al [9] derived the plenoptic spectrum in terms of $l_{x}(x, \bar{v})$ and determined properties based on 


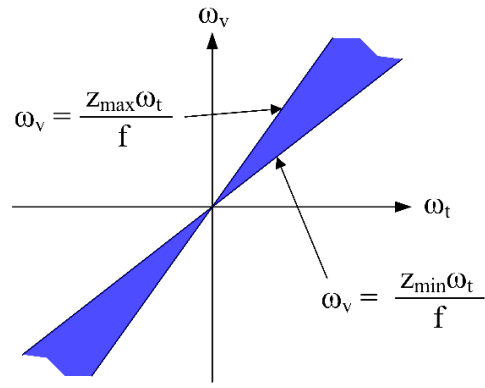

(a)

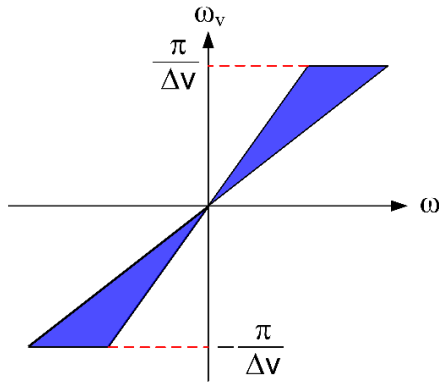

(b)

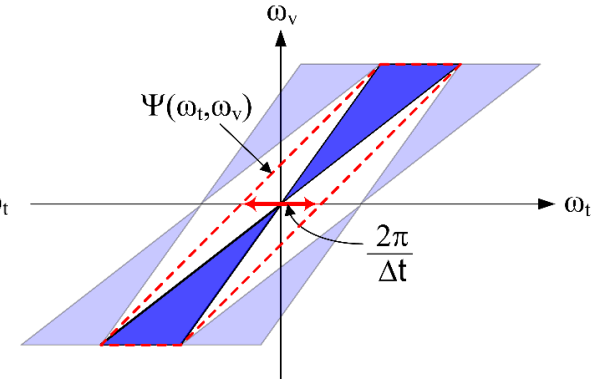

(c)

Fig. 5. Diagrams of the plenoptic spectrum: (a) the plenoptic spectrum bounded between $\omega_{v}=\omega_{t} z_{\min } / f$ and $\omega_{v}=\omega_{t} z_{\max } / f$. (b) the 'Bow-tie' shaped plenoptic spectrum caused by the pixel resolution, $\Delta v$, inducing lowpass filtering in $\omega_{v}$. (c) the optimal packing for the sampled plenoptic spectrum, where $\Delta t$ is the camera spacing. Note that $\Psi\left(\omega_{t}, \omega_{v}\right)$ is the reconstruction filter required for perfect reconstruction.

the behaviour of $l_{x}(x, \bar{v})$. Their expression for the plenoptic spectrum is obtained as follows: starting from its definition,

$$
P\left(\omega_{t}, \omega_{v}\right)=\int_{-\infty}^{\infty} \int_{-\infty}^{\infty} p(t, v) e^{-j\left(\omega_{t} t+\omega_{v} v\right)} d t d v
$$

the integration variables are changed using (1) and $\bar{v}=v / f$, which results in a Jacobian of $\left(1-z^{\prime}(x) \bar{v}\right) f$. Consequently the following is obtained

$$
\begin{aligned}
P\left(\omega_{t}, \omega_{v}\right)= & \int_{-\infty}^{\infty} \int_{-\infty}^{\infty} l_{x}(x, \bar{v}) e^{-j\left(\omega_{t}(x-z(x) \bar{v})+\omega_{v} f \bar{v}\right)} \\
& \cdot\left(1-z^{\prime}(x) \bar{v}\right) f d x d \bar{v} .
\end{aligned}
$$

Now, by defining $h(x, \bar{v})=l_{x}(x, \bar{v})\left(1-z^{\prime}(x) \bar{v}\right)$ and $L_{x}\left(x, \omega_{v}\right)=\mathcal{F}_{\bar{v}}\left\{l_{x}(x, \bar{v})\right\}$, where $\mathcal{F}_{\bar{v}}$ is the Fourier transform operator in $\bar{v}$, the following can be defined

$$
\begin{aligned}
H\left(x, \omega_{v}\right) & =\int_{-\infty}^{\infty} h(x, \bar{v}) e^{-j \omega_{v} \bar{v}} d \bar{v} \\
& =L_{x}\left(x, \omega_{v}\right)-j z^{\prime}(x) \frac{\partial L_{x}\left(x, \omega_{v}\right)}{\partial \omega_{v}} .
\end{aligned}
$$

Finally, by applying the above to (6), the following general equation for the plenoptic spectrum, independent of the scene's geometry, is obtained:

$$
P\left(\omega_{t}, \omega_{v}\right)=f \int_{-\infty}^{\infty} H\left(x, \omega_{v} f-z(x) \omega_{t}\right) e^{-j \omega_{t} x} d x .
$$

Now, by using the fact that $l_{x}(x, \bar{v})=l_{x}(x)$ for a Lambertian scene, [9] showed that in the frequency domain the following is true

$$
L_{x}\left(x, \omega_{v}\right)=0, \quad \text { if } \omega_{v} \neq 0,
$$

which leads to

$$
P\left(\omega_{t}, \omega_{v}\right)=0, \text { except when } \omega_{v} f=z(x) \omega_{t} .
$$

Therefore, as $z(x) \in\left[z_{\min }, z_{\max }\right]$, they showed that the plenoptic spectrum is precisely bounded by lines relating to the minimum and maximum depths of the scene, see Fig. 5(a).

\section{B. Uniform Sampling and Reconstruction}

Assuming a Lambertian scene, the plenoptic spectrum shown in Fig. 5(a) is bandlimited if lowpass filtering is applied in $\omega_{v}$. Such lowpass filtering occurs due to the finite pixel resolution, $\Delta v$, of the cameras. Therefore the plenoptic spectrum is bandlimited in $\omega_{v}$ at $\pi / \Delta v$, which results in the 'bow-tie' shaped spectrum shown in Fig. 5(b). Based on this shape, Chai et al [7] proposed that the replicated spectra should be packed as shown in Fig. 5(c) at critical sampling. To achieve this packing, without overlap occurring, they derive the following maximum camera spacing ${ }^{3}$

$$
\Delta t_{\mathrm{C}}=\frac{2 \pi z_{\max } z_{\min }}{\Omega_{v} f\left(z_{\max }-z_{\min }\right)},
$$

where $\Omega_{v}$ is the maximum frequency in $\omega_{v}$ (in the worse case it is equal to $\pi / \Delta v)$. The replicated spectra are removed using a skewed reconstruction filter, $\Psi\left(\omega_{t}, \omega_{v}\right)$, shown in Fig. 5(c) with a dashed line. The frequency support, $\mathcal{R}_{\psi}$, of this skewed filter is

$$
\left\{\omega_{t}, \omega_{v}: \omega_{v} \in\left[-\Omega_{v}, \Omega_{v}\right], \omega_{t} \in\left[\frac{\omega_{v}}{\eta}-\frac{\pi}{\Delta t}, \frac{\omega_{v}}{\eta}+\frac{\pi}{\Delta t}\right]\right\}
$$

where $\eta$ defines its skew. In [7] the skew is chosen as

$$
\eta_{\mathrm{C}}=2 \frac{z_{\max } z_{\min }}{\left(z_{\max }+z_{\min }\right) f} \text {. }
$$

\section{Plenoptic Spectral Analysis using Finite-View CAMERAS}

In Section III-A, we covered the derivation of the plenoptic spectrum presented in [9]. This derivation required the scene surface to be restricted by a no-occlusion constraint. This constraint is given by (2) and depends on the camera's field of view; if the field of view is finite then the scene depth can vary provided its gradient obeys the constraint. However, if the camera's field of view is not restricted then the scene surface must be flat and perpendicular to the camera's optical axis. This observation implies that the spectrum in (8) is only valid for scenes without depth variation as the spectral analysis does not limit the camera's field of view.

\footnotetext{
${ }^{3}$ Note that the subscript $\mathrm{C}$, short for Chai, is used to indicate that the result was presented in [7].
} 


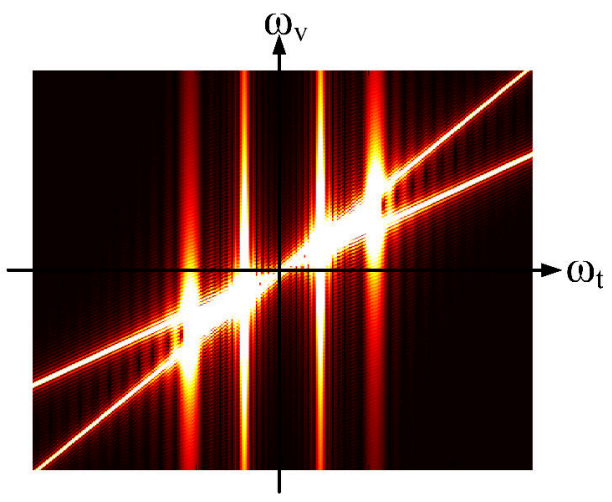

(a)

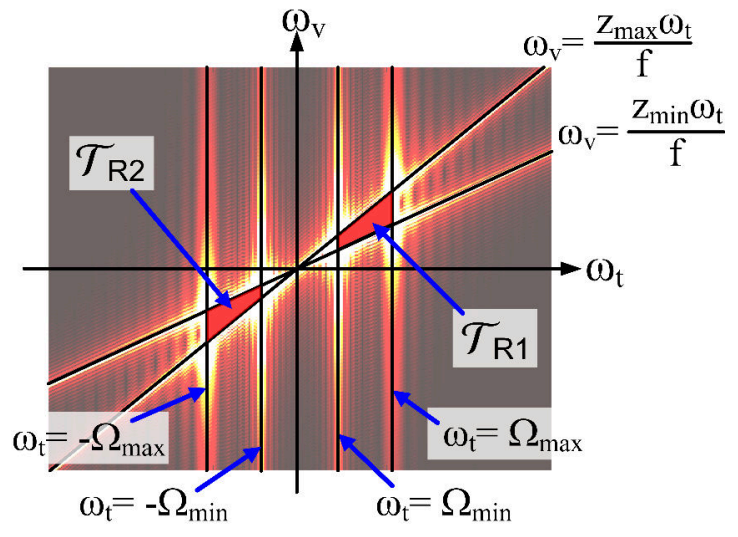

(b)

Fig. 6. Diagram (a) shows the magnitude plenoptic spectrum for a slanted plane with a texture signal $g(s)=\cos \left(\omega_{s} s\right)$. The same spectrum is shown in (b) but with the main characteristics of the spectrum superimposed. These characteristics comprise two quadrilateral regions bounded by six lines.

In view of this observation, we now examine the spectrum of the plenoptic function when the following two constraints are imposed: (i) cameras with finite field of view (FFV) and (ii) finite scene width (FSW). Starting from the plenoptic spectrum in (6), we assume a Lambertian scene, hence $l_{x}(x, \bar{v})=l_{x}(x)$, and impose our two constraints to obtain

$$
\begin{aligned}
P\left(\omega_{t}, \omega_{v}\right)= & \int_{x=x_{1}}^{x=x_{2}} l_{x}(x) e^{-j \omega_{t} x} \int_{\bar{v}=-\bar{v}_{m}}^{\bar{v}=\bar{v}_{m}}\left(1-z^{\prime}(x) \bar{v}\right) \\
& \cdot f e^{-j\left(\omega_{v} f-z(x) \omega_{t}\right) \bar{v}} d \bar{v} d x,
\end{aligned}
$$

where $\bar{v} \in\left[-\bar{v}_{m}, \bar{v}_{m}\right]$ is the FFV constraint, with $\bar{v}_{m}=v_{m} / f$, and $x \in\left[x_{1}, x_{2}\right]$ is the FSW constraint. Solving the integral in $\bar{v}$ the plenoptic spectrum becomes

$$
\begin{gathered}
P\left(\omega_{t}, \omega_{v}\right)=2 v_{m} \int_{x=x_{1}}^{x=x_{2}} l_{x}(x) \operatorname{sinc}\left(\omega_{I}\right) e^{-j \omega_{t} x} d x \\
-2 j v_{m} \bar{v}_{m} \int_{x=x_{1}}^{x=x_{2}} l_{x}(x) z^{\prime}(x) \operatorname{sinc}^{\prime}\left(\omega_{I}\right) e^{-j \omega_{t} x} d x,
\end{gathered}
$$

where $\operatorname{sinc}^{\prime}\left(\omega_{I}\right)$ is the first derivative of the sinc function ${ }^{4}$, and $\omega_{I}$ depends on $x$ as follows

$$
\omega_{I}=\omega_{v} v_{m}-z(x) \omega_{t} \bar{v}_{m} .
$$

This expression indicates that the two constraints cause spectral spreading in the plenoptic spectrum. In the following discussion, we shall analyse this spectral spreading for the specific case of the plenoptic spectrum for a slanted plane with bandlimited texture.

\section{A. Plenoptic Spectrum for a Slanted Plane}

Using the framework presented in [8], [9], the geometry of a slanted plane is described as follows

$$
\mathcal{G}_{s}=\left\{\begin{array}{l}
z(x)=\left(x-x_{1}\right) \tan (\phi)+z_{1} \text { for } x \in\left[x_{1}, x_{2}\right] \\
x(s)=s \cos (\phi)+x_{1} \text { for } s \in[0, T]
\end{array}\right.
$$

${ }^{4}$ We define $\operatorname{sinc}(\alpha)=\sin (\alpha) / \alpha$ for $\alpha \in \mathbb{R}$, thus $\operatorname{sinc}^{\prime}(\alpha)=\cos (\alpha) / \alpha-$ $\sin (\alpha) / \alpha^{2}$. where $\phi$ is the angle between the plane and the $x$-axis, $T$ is the length of the plane and $z\left(x_{2}\right)=z_{2}$. Therefore, the curvilinear coordinate, $s$, begins at $\left(x_{1}, z_{1}\right)$ and ends at $\left(x_{2}, z_{2}\right)$. A diagram of this geometric description is shown in Fig. 4. Without loss of generality, we shall assume $\phi \geq 0$ in this paper thus $z_{\max }=z_{2}$ is the maximum depth of the plane and $z_{\min }=z_{1}$ is the minimum depth of the plane. We also impose the constraint (2) in order to avoid self-occlusion. Therefore, from (2) and (16), we have the following bound

$$
0 \leq \phi<\tan ^{-1}\left(\frac{f}{v_{m}}\right) .
$$

Given the geometric description, we now define the bandlimited texture pasted to its surface. Similar to [8], [9], we assume this texture is real-valued with a discrete spectrum. Accordingly, we define the texture signal as a finite sum of complex exponentials and their conjugates:

$$
g(s)=\sum_{k=-K}^{K} \beta_{k} e^{j \omega_{k} s}
$$

where $\beta_{k}=\beta_{-k}^{*}$ is the coefficient for the $k$ th exponential, $\omega_{k}=-\omega_{-k}$ is the frequency of $k$ th exponential and $K \in \mathbb{N}$. Lastly, we define $\omega_{s}=\max _{k}\left\{\omega_{k}\right\}$ as the maximum frequency of the texture signal.

Given this complete description of the scene, we determine its plenoptic spectrum as follows. Using (16) and (18), we change the variable of integration in (15) from $x$ to the curvilinear coordinate $s$, and switch to the texture signal, i.e. $l(s)=g(s)$, to give

$$
\begin{aligned}
& P_{S}\left(\omega_{t}, \omega_{v}\right)=M_{1} \int_{0}^{T} g(s) \operatorname{sinc}\left(\hat{\omega}_{I}\right) e^{-j \omega_{t} \cos (\phi) s} \cos (\phi) d s \\
& -j M_{1} \bar{v}_{m} \tan (\phi) \int_{0}^{T} g(s) \operatorname{sinc}^{\prime}\left(\hat{\omega}_{I}\right) e^{-j \omega_{t} \cos (\phi) s} \cos (\phi) d s
\end{aligned}
$$

where $\quad \hat{\omega}_{I}=\omega_{v} v_{m}-\left(s \sin (\phi)+z_{\min }\right) \bar{v}_{m} \omega_{t}$,

$$
z^{\prime}(x)=\tan (\phi), \quad \text { and } \quad M_{1}=2 v_{m} e^{-j \omega_{t} x_{1}} .
$$




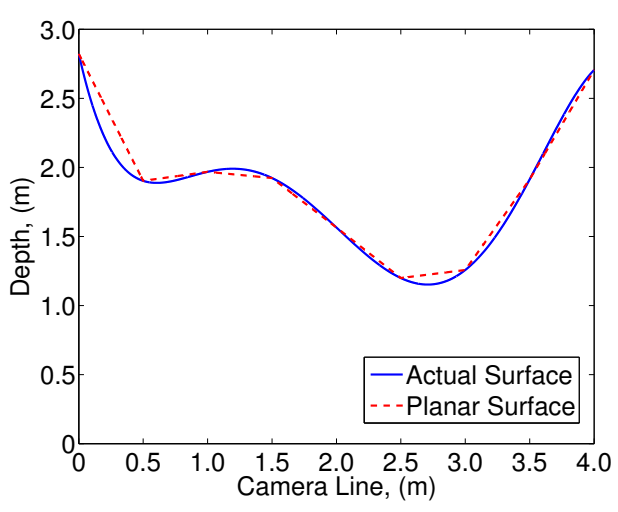

(a) Scene Geometry

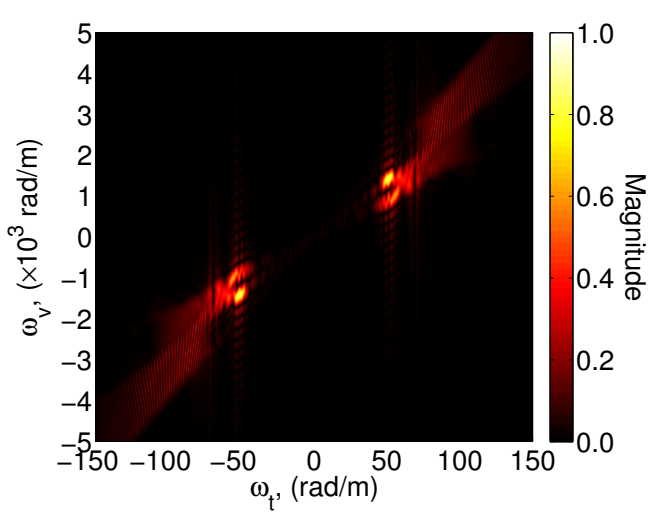

(c) $\left|P_{p}\left(\omega_{t}, \omega_{v}\right)\right|$

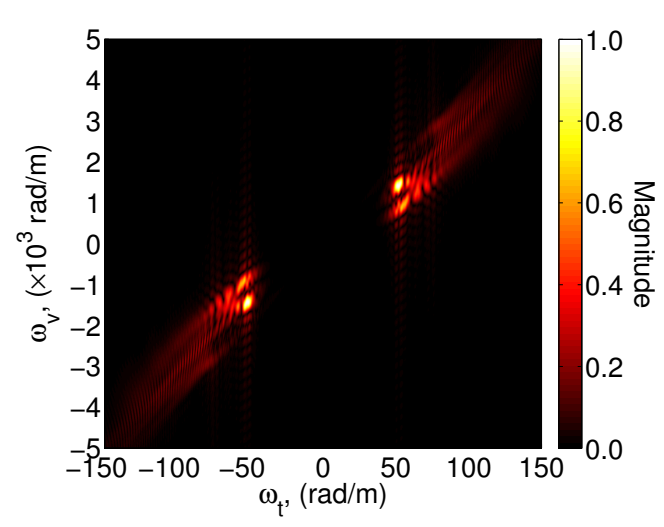

(b) $\left|P_{a}\left(\omega_{t}, \omega_{v}\right)\right|$

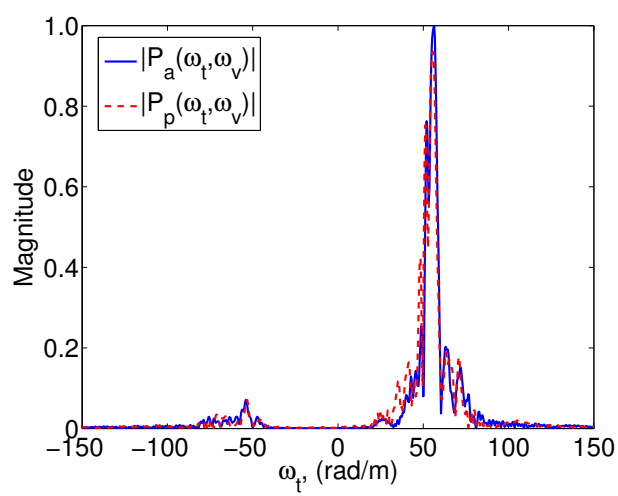

(d) Slice of the Plenoptic Spectra at $\omega_{v}=1 \times 10^{3} \mathrm{rad} / \mathrm{m}$

Fig. 7. Comparison of the plenoptic spectrum for a smoothly varying surface, $P_{a}\left(\omega_{t}, \omega_{v}\right)$, and the spectrum generated by approximating the surface with slanted planes, $P_{p}\left(\omega_{t}, \omega_{v}\right)$. Graph (a) illustrates the smooth surface and its planar approximation using 8 planes uniformly spaced along the $x$-axis. Both scenes have a texture $g(s)=\cos \left(\omega_{s} s\right)$. Graph (b) shows the magnitude spectrum of $P_{a}\left(\omega_{t}, \omega_{v}\right)$ and graph (c) shows the equivalent for $P_{p}\left(\omega_{t}, \omega_{v}\right)$. Graph (d) compares a 1D slice of both plenoptic spectra at a fixed $\omega_{v}=1 \times 10^{3} \mathrm{rad} / \mathrm{m}$.

Now, as we show in Appendix A, if $g(s)=e^{j \omega_{s} s}$ then the plenoptic spectrum has a closed-form expression. Therefore, using the linearity of the Fourier transform, we can obtain an exact expression when using (18) as texture. Before presenting this expression, we define, for convenience, the following three quantities

$$
\begin{aligned}
& a=\omega_{v} v_{m}-\omega_{t} z_{\max } \bar{v}_{m}, \\
& b=\omega_{v} v_{m}-\omega_{t} z_{\min } \bar{v}_{m}, \\
& c_{k}=\frac{\omega_{k}-\omega_{t} \cos (\phi)}{\sin (\phi) \omega_{t} \bar{v}_{m}} .
\end{aligned}
$$

The spectrum when $\omega_{t} \neq 0$ is

$$
\begin{gathered}
P_{S}\left(\omega_{t}, \omega_{v}\right)=\sum_{k=-K}^{K} \beta_{k}\left(\frac { j 2 v _ { m } } { \omega _ { t } } \left[\operatorname{sinc}(a) e^{-j T\left(\omega_{t} \cos (\phi)-\omega_{k}\right)}\right.\right. \\
-\operatorname{sinc}(b)]+\frac{j \omega_{k} f}{\sin (\phi) \omega_{t}^{2}}\left[\zeta\left\{j b\left(c_{k}-1\right)\right\}-\zeta\left\{j a\left(c_{k}-1\right)\right\}\right. \\
\left.\left.-\zeta\left\{j b\left(c_{k}+1\right)\right\}+\zeta\left\{j a\left(c_{k}+1\right)\right\}\right] e^{j b c_{k}}\right) e^{-j \omega_{t} x_{1}},
\end{gathered}
$$

where $\zeta$ is

$$
\zeta\{j \alpha\}= \begin{cases}\mathrm{E}_{1}(j \alpha)+\ln |\alpha|+j \frac{\pi}{2}+\gamma & \text { if } \alpha>0, \\ \mathrm{E}_{1}^{*}(j|\alpha|)+\ln |\alpha|-j \frac{\pi}{2}+\gamma & \text { if } \alpha<0, \\ 0 & \text { if } \alpha=0,\end{cases}
$$

$\alpha \in \mathbb{R}, \gamma$ is Euler's constant, $\mathrm{E}_{1}(j \alpha)$ is the exponential integral (see [27]) and $\mathrm{E}_{1}^{*}(j \alpha)$ is its complex conjugate. For the special case when $\omega_{t}=0$, the plenoptic spectrum is

$$
\begin{gathered}
P_{S}\left(0, \omega_{v}\right)=\sum_{k=-K}^{K} 2 \beta_{k} v_{m} T \operatorname{sinc}\left(\frac{\omega_{k} T}{2}\right)\left[\cos (\phi) \operatorname{sinc}\left(\omega_{v} v_{m}\right)\right. \\
\left.-j \frac{\sin (\phi) v_{m}}{f} \operatorname{sinc}^{\prime}\left(\omega_{v} v_{m}\right)\right] e^{j \omega_{k} \frac{T}{2}} .
\end{gathered}
$$

Using the expression in (21), Fig. 6(a) shows the magnitude of the plenoptic spectrum corresponding to a slanted plane with a texture signal $g(s)=\cos \left(\omega_{s} s\right)$.

\section{B. Behaviour of the Plenoptic Spectrum for a Slanted Plane}

Using the closed-form expression in (21), we can model the essential structure of the plenoptic spectrum for a slanted plane with real-valued texture. This model is illustrated in Fig. 6(b) for a texture signal $g(s)=\cos \left(\omega_{s} s\right)$. It comprises two quadrilateral regions, $\mathcal{T}_{R 1}$ and $\mathcal{T}_{R 2}$, which contain most of the spectral energy and are defined by six lines. The first two lines are diagonal and relate to the maximum and minimum depths of the slanted plane, i.e.

$$
\omega_{v}=\omega_{t} \frac{z_{\max }}{f} \text { and } \omega_{v}=\omega_{t} \frac{z_{\min }}{f} .
$$


These lines correspond respectively to the conditions $a=0$ and $b=0$ in (20). The other four lines are vertical and relate to the modulation of the texture signal when projected into the image plane. Using the rotational symmetry of the plenoptic spectrum, these four lines form two pairs $\omega_{t}= \pm \Omega_{\max }$ and $\omega_{t}= \pm \Omega_{\min }$. The quantity $\Omega_{\max }$ represents the maximum frequency of the modulated texture signal and $\Omega_{\min }$ represents its minimum ${ }^{5}$; they are defined as follows

$$
\begin{gathered}
\Omega_{\max }=\frac{\omega_{s}}{\cos (\phi)-|\sin (\phi)| \bar{v}_{m}} \\
\text { and } \Omega_{\min }=\frac{\min _{k}\left\{\omega_{k}\right\}}{\cos (\phi)+|\sin (\phi)| \bar{v}_{m}},
\end{gathered}
$$

and correspond to $c_{k}+1=0$, when $\omega_{k}=\omega_{s}$, and $c_{k}-1=0$, when $\omega_{k}=\min _{k}\left\{\omega_{k}\right\}$, in (20), respectively.

Using this model, we are now able to examine the behaviour of the plenoptic spectrum when aspects of the scene vary. Specifically, the slant of the plane, $\phi$, determines the gradient difference between the two diagonal lines in Fig. 6(b) since $z_{\max }-z_{\min }=T|\sin (\phi)|$. Moreover, the overall size of the quadrilateral regions, $\mathcal{T}_{R 1}$ and $\mathcal{T}_{R 2}$, changes with $\phi$. For example an increase in $\phi$ expands the two regions since $\Omega_{\max }$ increases and $\Omega_{\min }$ decreases. On the other hand, for the special case of $\phi=0$, these regions disappear entirely as $\Omega_{\max }=\Omega_{\min }$ and $z_{\max }=z_{\min }$. The quadrilateral regions also expand when the maximum frequency of the texture signal increases because $\Omega_{\max }$ is proportional to $\omega_{s}$.

\section{Determining the Plenoptic Spectrum of a Smoothly Varying Surface}

The linearity of the Fourier transform allows the result in (21) to be extended to scenes comprising multiple slanted planes provided that the no-occlusion condition is met. Therefore, given a scene that has a smoothly varying surface with no-occlusions and bandlimited texture, we can approximate the scene with a set of slanted planes and use (21), evaluated for each plane, to determine its plenoptic spectrum. The accuracy of this plenoptic spectrum will depend on the number of planes used to approximate the surface.

To demonstrate using (21) as a proxy, we compare, in Fig. 7 , the plenoptic spectrum for a smooth surface to the spectrum obtained using slanted planes. The smooth surface, shown in Fig. 7(a) with a solid blue line, is generated using Legendre polynomials up to degree 5 and has a texture $g(s)=\cos \left(\omega_{s} s\right)$. Its magnitude plenoptic spectrum, $\left|P_{a}\left(\omega_{t}, \omega_{v}\right)\right|$, is calculated numerically and illustrated in Fig. 7(b). We now approximate this surface with 8 slanted planes, as shown in Fig. 7(a) with a dashed red line. Note that the planes are uniformly spaced along the $x$-axis. Using this approximation, we obtain analytically the planar plenoptic spectrum, $\left|P_{p}\left(\omega_{t}, \omega_{v}\right)\right|$, shown in Fig. 7(c) $)^{6}$. This planar plenoptic spectrum has a PSNR $=27 \mathrm{~dB}$ when compared to $P_{a}\left(\omega_{t}, \omega_{v}\right)$ thus it represents a good proxy for the original. Notice that a comparison of

\footnotetext{
${ }^{5}$ In [9] this modulation of the texture signal is shown to be equivalent to time-warping the signal with a scene dependent warping function.

${ }^{6}$ Note that all the spectra in Fig. 7 are normalized with respect to $\left|P_{a}\left(\omega_{t}, \omega_{v}\right)\right|$.
}

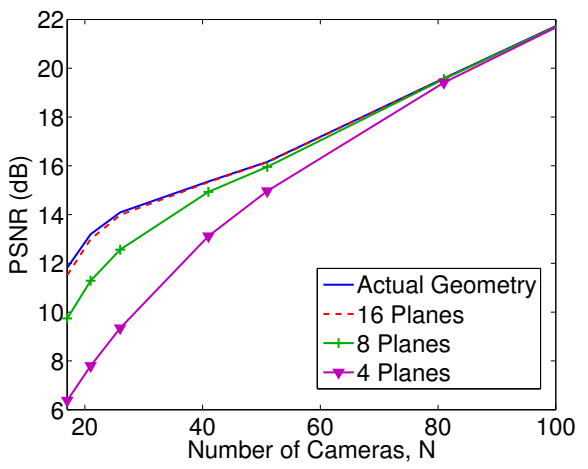

Fig. 8. Graph examining the reconstruction of the EPI generated by the smooth surface in Fig. 7(a). The EPI is reconstructed from uniform samples using the actual scene geometry; a 16 plane approximation; a 8 plane approximation; and a 4 plane approximation.

the 1D slice of the two plenoptic spectra, obtained by fixing $\omega_{v}=1 \times 10^{3} \mathrm{rad} / \mathrm{m}$, is shown in Fig. 7(d).

To further gauge the effect of this planar approximation, we examine the reconstruction of the EPI relating to the scene in Fig. 7(a). Given uniform samples of this EPI, we reconstruct it using three planar approximations that vary in coarseness from 4 planes to 8 planes, shown in Fig 7(a), and finally 16 planes. The results of these differing reconstructions, along with the reconstruction achieved using the actual surface, are shown in Fig. 8. The figure shows that the reconstruction improves as the accuracy of the approximation increase and that the results achieved using 16 planes are almost equivalent to those obtained using the actual surface.

\section{Plenoptic Sampling using the Essential BANDWIDTH}

From (21), we observed that the plenoptic spectrum for a slanted plane is band-unlimited. We therefore propose utilising the concept of the essential bandwidth [28] when sampling its plenoptic function. The essential bandwidth is a region in frequency that contains a large fraction of the signal's energy. In [28], it was observed that the main lobe of a 1D sinc function contains $90 \%$ of its energy. If we extend this to a $2 \mathrm{D}$ frequency domain then the main lobe of a $2 \mathrm{D}$ sinc function will contain at least $81 \%$ of its energy. Therefore, we define the essential bandwidth to be a compact region in the frequency domain that is symmetrical around the origin and that contains at least $81 \%$ of the plenoptic spectrum's energy. We will then assume the plenoptic spectrum is approximately bandlimited to its essential bandwidth and determine the Nyquist sampling rate accordingly.

The essential bandwidth for the plenoptic function has also been studied in [9]. In their analysis, one of the dimensions of the plenoptic function is fixed, either $t$ or $v$, and the resulting essential bandwidth is determined for an unconstrained plenoptic function. In contrast, we are interested in determining a non-separable $2 \mathrm{D}$ essential bandwidth in the $\left(\omega_{t}, \omega_{v}\right)$-domain that takes into account both FSW and FFV. 


\section{A. Determining the Essential Bandwidth}

From the structural model of the plenoptic spectrum presented in Section IV-B, we observe that the spectral energy is concentrated in the two quadrilateral regions, $\mathcal{T}_{R 1}$ and $\mathcal{T}_{R 2}$, shown in Fig. 6(b). Thus it is natural to design a bandwidth that contains these regions. Accordingly, we propose a parallelogram shape for the essential bandwidth, $B_{S}$, of the slanted plane as illustrated in Fig. 9(a). The figure shows that the parallelogram is defined by four parameters: $\Omega_{v}$, the maximum value in $\omega_{v} ; \Omega_{t}$, the maximum value in $\omega_{t} ; A$, the width of the region in $\omega_{t}$; and $z_{\mathrm{G}} / f$, a parameter that controls the skew of the parallelogram relative to the $\omega_{v}$-axis. We obtain these parameters by analyzing the bandwidth of the plenoptic spectrum as follows.

We start by extending our plenoptic spectral analysis to include a general texture signal, $g(s)$, that is bandlimited to $\omega_{s}$. To achieve this, we return to the expression in (19) and combine the FSW condition with the texture signal, $g(s)$, to give the following function

$$
h(s)=g(s) \text { if } s \in[0, T] \text { and } h(s)=0 \text { otherwise. }
$$

We then define $H(\Omega)$ as the Fourier transform of $h(s)$ and $\Omega$ as its frequency variable. Now, by expressing $h(s)$ as an inverse Fourier transform of $H(\Omega)$, we can obtain the following expression from (19)

$$
\begin{aligned}
P_{S}\left(\omega_{t}, \omega_{v}\right)= & M_{2} \int_{-\infty}^{\infty} H(\Omega) \frac{f \Omega}{\sin (\phi) \omega_{t}^{2}} \operatorname{rect}\left(\frac{\Omega-\omega_{t} \cos (\phi)}{2 \sin (\phi) \bar{v}_{m} \omega_{t}}\right) \\
& \cdot e^{-j \Omega\left(\frac{z_{\min }}{\sin (\phi)}-\frac{\omega_{v} f}{\sin (\phi) \omega_{t}}\right)} d \Omega
\end{aligned}
$$

where

$$
M_{2}=e^{-j \omega_{t}\left(x_{1}-\frac{z_{\min }}{\tan (\phi)}\right)} e^{-j \omega_{v}\left(\frac{f}{\tan (\phi)}\right)} .
$$

The derivation of this expression is shown in Appendix B. Given (24), we now examine its bandwidth to obtain the parameters of $B_{S}$.

We first consider the line defined by $\omega_{v}=\omega_{t} z_{\max } / f$ that corresponds to the upper boundary of region $\mathcal{T}_{R 1}$ in Fig. 6(b). The 1D plenoptic spectrum along this line, $P_{1}\left(\omega_{t}, \omega_{t} z_{\max } / f\right)$, is illustrated in Fig. 9(b). The essential bandwidth for this 1D plenoptic spectrum, indicated with dashed lines in Fig. 9(b), is found in Appendix $\mathrm{C}$ to be

$$
B_{t}=\left\{\omega_{t}:\left|\omega_{t}\right| \leq \frac{1}{\cos (\phi)-\bar{v}_{m}|\sin (\phi)|}\left(\omega_{s}+\frac{2 \pi}{T}\right)\right\}
$$

Using this bandwidth, we set the maximum value of $\omega_{t}$ equal to the maximum frequency in $B_{t}$ and obtain

$$
\Omega_{t}=\frac{1}{\cos (\phi)-\bar{v}_{m}|\sin (\phi)|}\left(\omega_{s}+\frac{2 \pi}{T}\right) .
$$

Using (27), we now determine the equivalent parameter in $\omega_{v}$. We start by setting $\omega_{t}=\Omega_{t}$ in (24) to obtain a vertical slice of the plenoptic spectrum shown in Fig. 9(c). The essential bandwidth of this vertical slice, indicated with dashed lines in
Fig. 9(c), is shown in Appendix D to be

$$
\begin{gathered}
B_{v}=\left\{\omega_{v}: \omega_{v} \in\left[\Omega_{t} \frac{z_{\min }}{f}-n(\phi, \bar{v}) \frac{\pi}{v_{m}},\right.\right. \\
\left.\left.\Omega_{t} \frac{z_{\max }}{f}+n(\phi, \bar{v}) \frac{\pi}{v_{m}}\right]\right\},
\end{gathered}
$$

where

$$
n\left(\phi, \bar{v}_{m}\right)=\frac{3 \cos ^{2}(\phi)+3.5\left(\bar{v}_{m} \sin (\phi)\right)^{2}}{3 \cos ^{2}(\phi)+\left(\bar{v}_{m} \sin (\phi)\right)^{2}} .
$$

Accordingly, similar to $\Omega_{t}$, we set the maximum value of $\omega_{v}$ equal to the maximum frequency in $B_{v}$ and obtain

$$
\Omega_{v}=\Omega_{t} \frac{z_{\max }}{f}+n\left(\phi, \bar{v}_{m}\right) \frac{\pi}{v_{m}} .
$$

Finally, we now determine the last two parameters. First, we note that for real-valued texture the essential bandwidth of the slanted plane has rotational symmetric around the origin. Therefore, using the shape of the parallelogram, we can formulate the following relationship when $\omega_{t}=\Omega_{t}$ and $\omega_{v}=\Omega_{v}$ :

$$
\Omega_{v}=\Omega_{t} \frac{z_{\max }}{f}+n\left(\phi, \bar{v}_{m}\right) \frac{\pi}{v_{m}}=\frac{z_{\mathrm{G}}}{f}\left(\Omega_{t}+\frac{A}{2}\right) .
$$

Next, we use the minimum frequency from $B_{v}$ and formulate a second relationship, which is

$$
\Omega_{t} \frac{z_{\max }}{f}-n\left(\phi, \bar{v}_{m}\right) \frac{\pi}{v_{m}}=\frac{z_{\mathrm{G}}}{f}\left(\Omega_{t}-\frac{A}{2}\right) .
$$

Therefore, by solving (31) and (32), we can obtain

$$
z_{\mathrm{G}}=\frac{z_{\max }+z_{\min }}{2}
$$

and

$$
A=\frac{\Delta z}{z_{\mathrm{G}}} \Omega_{t}+n\left(\phi, \bar{v}_{m}\right) \frac{2 \pi f}{v_{m} z_{\mathrm{G}}},
$$

where $\Delta z=z_{\max }-z_{\min }=T|\sin (\phi)|$ is the depth variation of the plane.

\section{B. Analysis of the Essential Bandwidth}

Having determined the essential bandwidth $B_{S}$, we now analyze the amount of energy the chosen region contains. The analysis is performed using a synthetic scene comprising a single slanted plane with bandlimited texture. From the behaviour of the plenoptic spectrum highlighted in Section IV-B, we observe that $\omega_{s}$ and $\phi$ have the greatest effect on the structure of the spectrum. As a result, we analyze the energy in $B_{S}$ as both $\omega_{s}$ and $\phi$ vary. The other scene parameters are fixed as follows: $z_{\min }=1.5 \mathrm{~m}, T=3.5 \mathrm{~m}$, and the cameras are defined with a focal length equal to $32 \mathrm{~mm}$ and a field of view $(\mathrm{FV})=40^{\circ}$. Using this geometry, we vary the angle $\phi$ from 0 to just below the limit imposed by (17), and $\omega_{s}$ from 0 to $600 \mathrm{rad} / \mathrm{m}$.

The results of the analysis are shown in Fig. 10. The figure presents a $2 \mathrm{D}$ plot of the percentage of energy inside $B_{S}$ as a function of $\omega_{s}$ and $\phi$. The results show that for all values of $\omega_{s}$ and $\phi$ our essential bandwidth $B_{S}$ always contains at least $89 \%$ of the energy of the plenoptic function. The figure also illustrates that our derivation of the essential bandwidth is conservative for larger values of $\omega_{s}$ and $\phi$. 


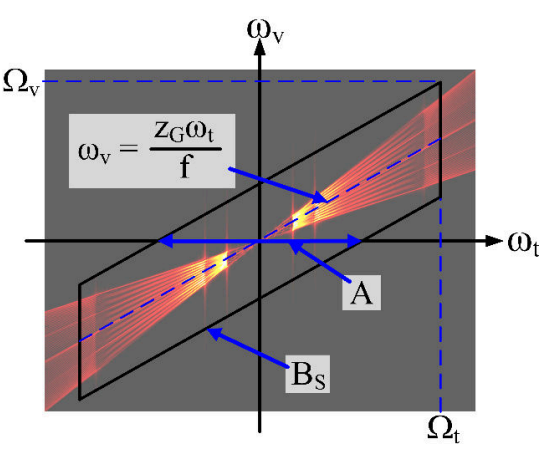

(a) $\left|P_{1}\left(\omega_{t}, \omega_{v}\right)\right|$

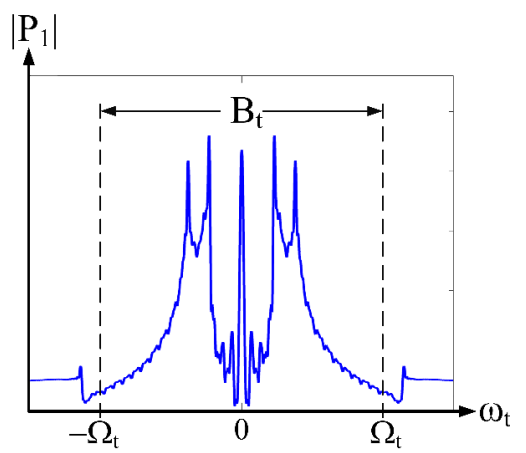

(b) $\left|P_{1}\left(\omega_{t}, \omega_{t} z_{\max } / f\right)\right|$

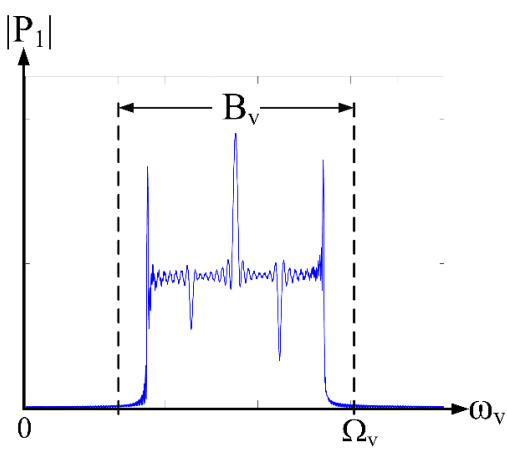

(c) $\left|P_{1}\left(\Omega_{t}, \omega_{v}\right)\right|$

Fig. 9. Diagram (a) illustrates the parametric essential bandwidth, $B_{S}$, superimposed on the plenoptic spectrum of a slanted plane with bandlimited texture, $P_{1}\left(\omega_{t}, \omega_{v}\right)$. Diagram (b) shows the 1D slice of $P_{1}\left(\omega_{t}, \omega_{v}\right)$ when $\omega_{v}=\omega_{t} z_{\max } / f$. The region $B_{t}$ represents its essential bandwidth and $\Omega_{t}$ represents the maximum frequency of $B_{t}$. Diagram (c) shows the 1D slice of $P_{1}\left(\omega_{t}, \omega_{v}\right)$ when $\omega_{t}=\Omega_{t}$. The region $B_{v}$ represents its essential bandwidth and $\Omega_{v}$ represents the maximum frequency of $B_{v}$.

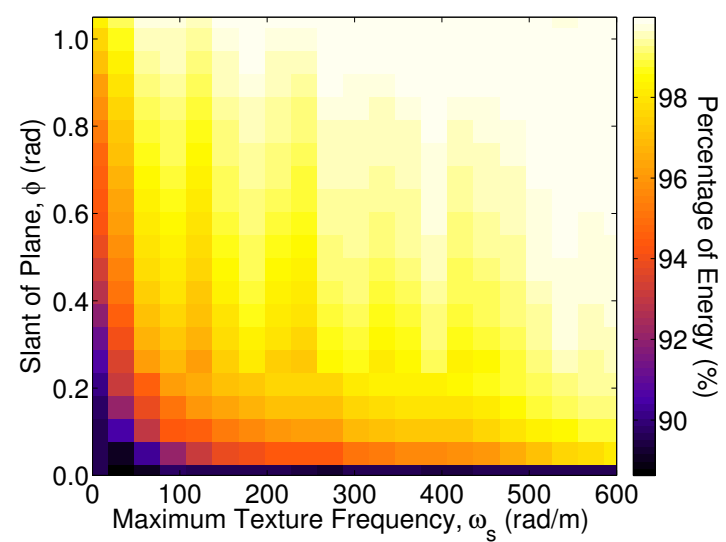

Fig. 10. Graph showing the percentage of the plenoptic spectrum's energy inside $B_{S}$ as a function of $\omega_{s}$ and $\phi$. The plenoptic spectrum corresponds to a slanted plane with bandlimited texture.

\section{Sampling and Reconstruction}

Given the essential bandwidth $B_{S}$, we now examine the sampling and reconstruction of the plenoptic function. From Section III-B, the Nyquist rate in uniform plenoptic sampling occurs when the replicated spectra are packed according to Fig. 5(c). Assuming the plenoptic function in question is bandlimited to $B_{S}$, this packing is achieved when

$$
\frac{2 \pi}{\Delta t}=A \text {. }
$$

Therefore, using (34), we obtain the following expression for the maximum camera spacing for a slanted plane

$$
\Delta t_{\mathrm{G}}=\frac{2 \pi}{A}=\frac{2 \pi z_{\mathrm{G}} v_{m}}{v_{m} \Omega_{t} \Delta z+2 \pi n\left(\phi, \bar{v}_{m}\right) f} .
$$

As we shall see in the next section, this new expression leads to a different Nyquist rate when compared to $\Delta t_{\mathrm{C}}$ from (11).

In terms of reconstruction, we still require the skewed filter defined in (12) to remove the unwanted replicated spectra. However, instead of the skew defined by (13), we use the skew of the essential bandwidth, thus we obtain the following expression for the filter skew

$$
\eta_{\mathrm{G}}=\frac{z_{\mathrm{G}}}{f}=\frac{z_{\max }+z_{\mathrm{min}}}{2 f} .
$$

The new reconstruction filter created by setting $\eta=\eta_{\mathrm{G}}$ in (12) is equivalent to rendering new images with a constant depth $z_{\mathrm{G}}$. Thus, in the case of a slanted plane, we propose rendering using the average depth of the scene rather than the inverse of the average disparity of the scene, $\eta_{\mathrm{C}} f$, presented in [7].

To conclude, by determining the essential bandwidth of a slanted plane, we are able to determine a new expression for the maximum camera spacing and a new reconstruction filter.

\section{High Dimensional Plenoptic Functions}

In this section, we briefly highlight how, under certain conditions, our analysis can be extended to higher dimensional plenoptic functions. The scene, in this case, is a 2D planar facet with a 2D texture signal, $g(s, r)$, pasted to its surface. The 3D EPI-volume is an extension of the EPI in which the cameras are still restricted to a camera line however they now capture 2D images $(v, w)$. In this situation, our spectral analysis and sampling results are still valid provided the depth of the planar facet in the $y$ direction is constant (i.e. the scene depth only varies with $x$ ) and the texture signal is separable such that $g(s, r)=g(s) g(r)$. The extension to the more general case however is not straightforward, in particular the 4D lightfield allows an extra degree of freedom when positioning the cameras.

\section{Simulation Results}

In this section we evaluate our proposed sampling results for the plenoptic function of a slanted plane. Specifically, we want to evaluate the filter skew $\eta_{\mathrm{G}}$, defined in (37), and the camera spacing $\Delta t_{\mathrm{G}}$, defined in (36). We perform this evaluation by sampling and reconstructing a plenoptic function generated by a synthetic scene in Section VI-A and a real scene in Section VI-B. In both cases the sampling is uniform (i.e. uniform camera placement) and reconstruction achieved using a skewed linear filter. Note that the purpose of this type of 


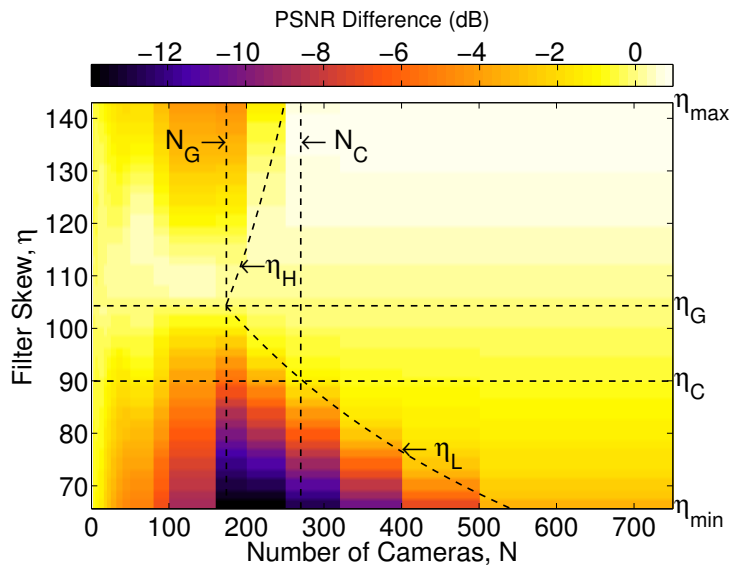

(a)

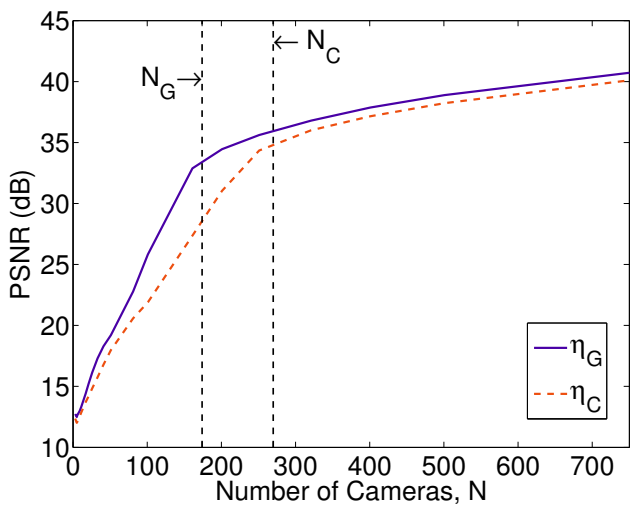

(b)

Fig. 11. Graphs examining the reconstruction of a synthetic EPI. In (a) the skew of the reconstruction filter varies from $\eta_{\min }=z_{\min } / f$ to $\eta_{\max }=z_{\max } / f$. The intensity in the image represents the difference in PSNR relative to the reconstruction achieved using $\eta_{\mathrm{G}}$. The lines $\eta_{\mathrm{L}}$ and $\eta_{\mathrm{H}}$ represent the lower and upper range of the filter skew defined in (38). In (b) the graph compares the absolute PSNR when the filter skew is $\eta_{\mathrm{G}}$ and $\eta_{\mathrm{C}}$. The quantities $N_{\mathrm{G}}$ and $N_{\mathrm{C}}$ are the number of cameras required to achieve $\Delta t_{\mathrm{G}}$ and $\Delta t_{\mathrm{C}}$, respectively.

reconstruction is to evaluate our filter skew $\eta_{\mathrm{G}}$ rather than as a method for view synthesis in IBR. This is not to say that the proposed theory has no place in competitive IBR algorithms; for example, layer-based IBR algorithms, such as [16], [17], currently use the theory from [7] to inform the number of the layers in the scene.

In the following analysis, the PSNR is measured over the entire plenoptic function and the Nyquist number of cameras predicted by (36) and (11) are marked as $N_{\mathrm{G}}$ and $N_{\mathrm{C}}$, respectively ${ }^{7}$.

\section{A. Synthetic Scenes}

To evaluate our results, we analyze the reconstruction of a synthetic EPI as the filter skew, $\eta$, varies from $\eta_{\min }=z_{\min } / f$ to $\eta_{\max }=z_{\max } / f$. The synthetic EPI correspond to a single slanted plane with bandlimited texture. The plane has the following parameters $\phi=45^{\circ}, \omega_{s}=125 \mathrm{rad} / \mathrm{m}, z_{\min }=2.1 \mathrm{~m}$ and $T=3.5 \mathrm{~m}$. The cameras are defined with a focal length equal to $32 \mathrm{~mm}$ and a $\mathrm{FV}=40^{\circ}$. The results of the analysis

\footnotetext{
${ }^{7}$ To calculate $N_{\mathrm{C}}$, we substitute the definition of $\Omega_{v}$ from (30) into the camera spacing defined in (11).
}

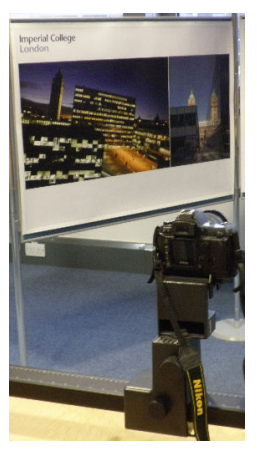

(a)

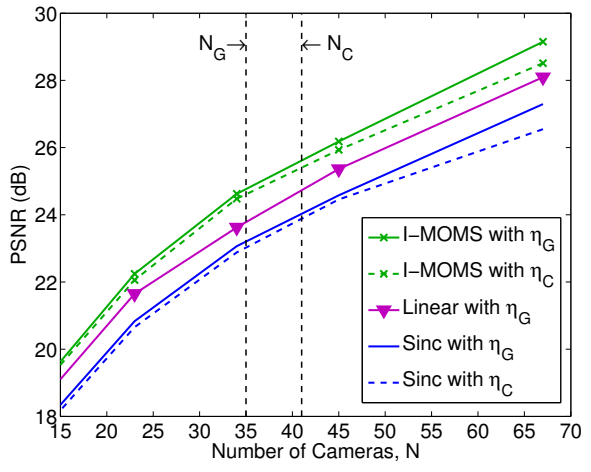

(b)
Fig. 12. Reconstructing the EPI-volume for a real scene: Diagram (a) illustrates the planar scene and the data acquisition set-up. The EPI-volume consists of 133 images spaced $1 \mathrm{~cm}$ apart and each image has 3008 by 1888 pixels. The cameras have a focal length of $70 \mathrm{~mm}$, which corresponds to a FV $=20^{\circ}$. Diagram (b) compares the reconstruction of the EPI-volume achieved using five different filters; a $3^{\text {rd }}$ order I-MOMS with filter skew $\eta_{\mathrm{G}}$; a $3^{\text {rd }}$ order I-MOMS with filter skew $\eta_{\mathrm{C}}$; a linear filter with skew $\eta_{\mathrm{G}}$; a sinc function with filter skew $\eta_{\mathrm{G}}$; and a sinc function with filter skew $\eta_{\mathrm{C}}$. The Nyquist number of samples required for $\Delta t_{\mathrm{G}}$ and $\Delta t_{\mathrm{C}}$ are indicated with $N_{\mathrm{G}}$ and $N_{\mathrm{C}}$, respectively.

are shown in Fig. 11. The first plot, Fig. 11(a), examines the reconstruction of the synthetic EPI as a function of the filter skew, $\eta$, and the number of cameras, $N$. The intensity in the plot represents the difference in PSNR when that reconstruction is compared to the reconstruction achieved using $\eta=\eta_{\mathrm{G}}$. In the second plot, Fig. 11(b), the absolute PSNR curves when using $\eta_{\mathrm{G}}$ and $\eta_{\mathrm{C}}$ are compared.

Fig. 11(a) shows that a skew of $\eta_{\mathrm{G}}$ gives the best reconstruction when the number of cameras is equal to or less than 250. However, when more cameras are available, the gain in performance is reduced until there is little distinction between the different filter skews. The reason for this behaviour can be seen by considering Fig. 9(a) and the support of the reconstruction filter defined in (12). If $\eta=\eta_{\mathrm{G}}$, the reconstruction filter comprises a band centered on the diagonal dashed line of slope $\eta_{\mathrm{G}}$ shown in Fig. 9(a) and the width of the band is proportional to the camera density. At the critical camera density, the width of this band equals that of the essential bandwidth parallelogram in the figure. When too few cameras are available (i.e. undersampling), the filter support is narrower than the essential bandwidth and the spectral energy captured by the filter depends strongly on the filter skew. When many cameras are available (i.e. oversampling), the filter support is wider and will include the entire essential bandwidth for filter skews in the range

$$
\eta \in\left[\frac{\left(\Omega_{t}+A / 2\right) \eta_{\mathrm{G}}}{\left(\Omega_{t}+\pi / \Delta t\right)}, \frac{\left(\Omega_{t}-A / 2\right) \eta_{\mathrm{G}}}{\left(\Omega_{t}-\pi / \Delta t\right)}\right] .
$$

The upper and lower limits of this range are marked respectively $\eta_{\mathrm{H}}$ and $\eta_{\mathrm{L}}$ in Fig. 11(a). Therefore, a skew of $\eta_{\mathrm{G}}$ represents the best choice when sampling at or below the Nyquist rate predicted by (36) but oversampling will compensate a poor choice of filter skew. Finally, the figures also highlight that the prediction of the Nyquist number of cameras, $N_{\mathrm{G}}$, is less conservative than that predicted in [7], $N_{\mathrm{C}}$. This relationship is reversed however when $\phi$ tends to 


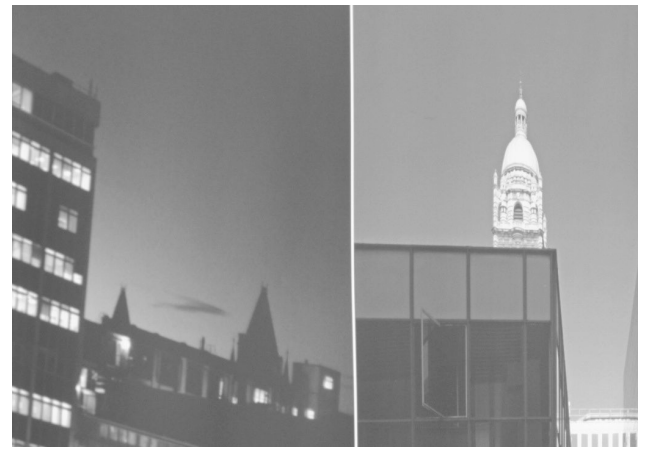

(a) Original

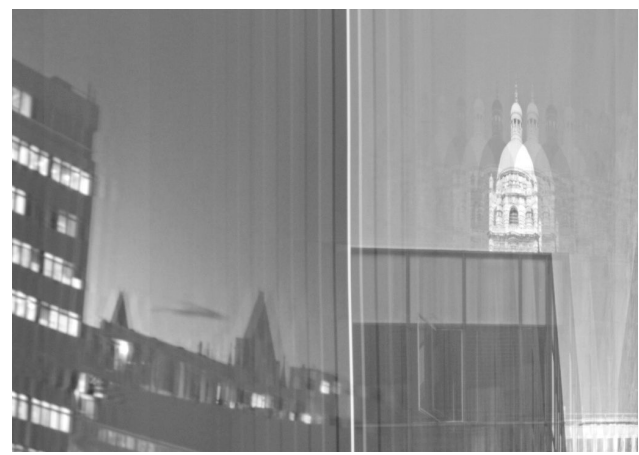

(c) Sinc Function with $\eta_{\mathrm{C}}$, PSNR $=22.4 \mathrm{~dB}$

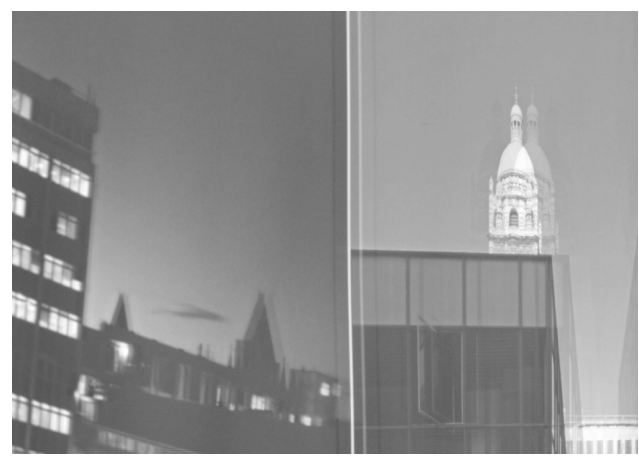

(e) I-MOMS with $\eta_{\mathrm{G}}$, PSNR $=23.2 \mathrm{~dB}$

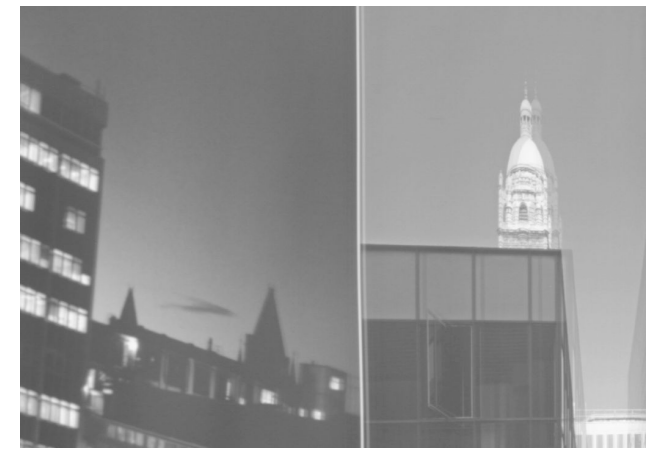

(b) Linear Filter with $\eta_{\mathrm{G}}$, PSNR $=23.9 \mathrm{~dB}$

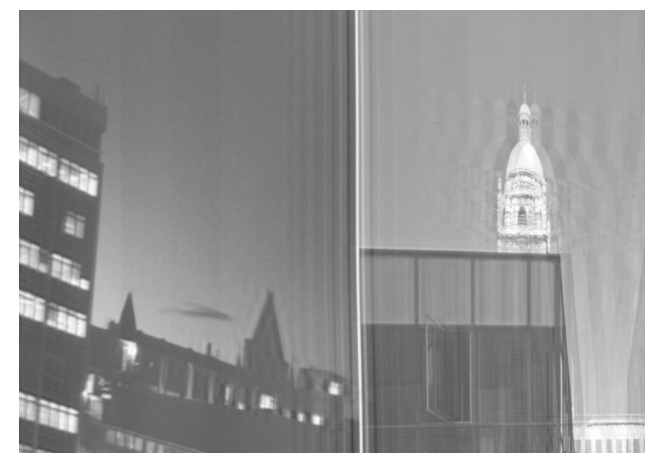

(d) Sinc Function with $\eta_{\mathrm{G}}$, PSNR $=23.6 \mathrm{~dB}$

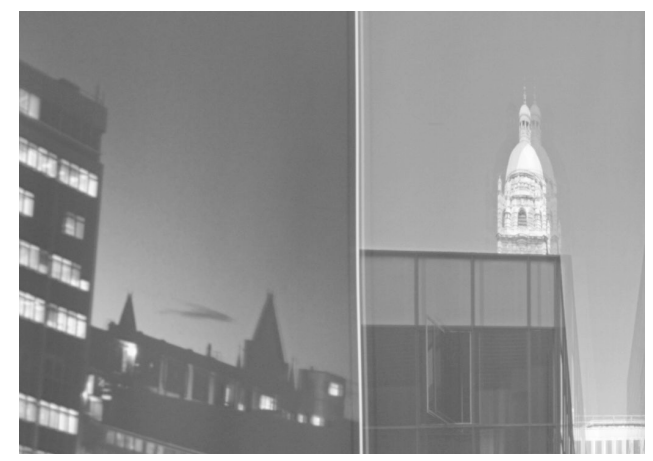

(f) I-MOMS with $\eta_{\mathrm{G}}$, PSNR $=24.2 \mathrm{~dB}$

Fig. 13. An example of a rendered image from the EPI-volume. The original is shown in (a), it's rendering using a linear filter with $\eta_{\mathrm{G}}$ in (b), using a sinc function with a skew $\eta_{\mathrm{C}}$ in (c), using a sinc function with a skew $\eta_{\mathrm{G}}$ in (d), using a $3^{\text {rd }}$ order I-MOMS with $\eta_{\mathrm{C}}$ in (e) and using a $3^{\text {rd }}$ order I-MOMS with a skew $\eta_{\mathrm{G}}$ in (f). Each image is rendered using 34 of the original images.

zero; the camera number $N_{\mathrm{C}}$ is equal to zero when $\phi=0$ whereas $N_{\mathrm{G}}$ tends to a constant that depends on the FFV of the cameras.

\section{B. Real Scene}

We now analyze the reconstruction of a 3D EPI-volume generated from a real scene comprising a single slanted plane, shown in Fig. 12(a). The EPI-volume is a simple extension of the EPI, in which the cameras are still restricted to a camera line, $t$, however they now capture 2D images $(v, w)$. For this analysis, the EPI-volume consists of 133 images, each 3008 by 1888 pixels in size, spaced $1 \mathrm{~cm}$ apart along the camera line. The process of acquiring the data is illustrated in Fig. 12(a). The images are acquired using a camera with a $70 \mathrm{~mm}$ focal length, which corresponds to a $\mathrm{FV}=20^{\circ}$. Notice that the real scene is constrained such that the depth only varies with $x$, hence the EPI-volume can be treated as a set of $2 \mathrm{D}$ EPIs stacked together.

Given the EPI-volume defined above, we analyze its reconstruction using five different filters. The first two filters have a frequency support equivalent to (12) but with different filter skews, $\eta_{\mathrm{G}}$ and $\eta_{\mathrm{C}}$. This means that each filter has an impulse response in the EPI-domain that is equivalent to a $2 \mathrm{D}$ skewed sinc function. Since the sinc function has infinite support and slow decay, its use is appropriate when reconstructing globally smooth signals. However, when dealing with piecewise smooth signals, like images, the sinc is less appropriate. For this reason, the remaining filters considered have compact support. Specifically, we use the $3^{\text {rd }}$ order Interpolating MaximalOrder-Minimal-Support (I-MOMS) proposed in [29] for the third and fourth filters. This function has the minimum compact support for a given accuracy or approximation order when 
interpolating. Similar to the sinc functions, the two I-MOMS filters have different skews, $\eta_{\mathrm{G}}$ and $\eta_{\mathrm{C}}$. Lastly, for comparison, we use a linear filter that interpolates along the EPI lines, assuming a constant gradient of $1 / \eta_{\mathrm{G}}$, to reconstruct the EPIvolume. $^{8}$

The results of this comparison are shown in Fig. 12(b). The figure shows that, for both the sinc functions and the I-MOMS filters, using a skew $\eta_{\mathrm{G}}$ gives a small but consistent improvement in PSNR over a skew of $\eta_{\mathrm{C}}$. As expected, the reconstruction of the EPI-volume is improved by using filters of compact support (i.e the linear filter or the I-MOMS filter). The best results are achieved using the $3^{\text {rd }}$ order I-MOMS filter; the gain in performance over the sinc functions is approximately $1 \mathrm{~dB}$ for all camera densities and approximately $0.8 \mathrm{~dB}$ when compared to the linear filter. To illustrate these results, Fig. 13 shows a section of an image rendered using each filter; the original is shown in Fig. 13(a); the rendering using the linear filter with $\eta_{\mathrm{G}}$ in Fig. 13(b); the rendering using the sinc function with $\eta_{\mathrm{C}}$ in Fig. 13(c); the rendering using the sinc function with $\eta_{\mathrm{G}}$ in Fig. 13(d); the rendering using the IMOMS with $\eta_{\mathrm{C}}$ in Fig. 13(e); and the rendering using the I-MOMS with $\eta_{\mathrm{G}}$ in Fig. 13(f). The PSNR of each rendered image is respectively $23.9 \mathrm{~dB}, 22.4 \mathrm{~dB}, 23.6 \mathrm{~dB}, 23.2 \mathrm{~dB}$ and $24.2 \mathrm{~dB}$

\section{CONCLUSION}

In this paper, we have studied the spectrum of the plenoptic function using a slanted plane as a building block for more sophisticated scenes. We performed the spectral analysis assuming the scene has a finite width and that the cameras have a finite field of view. Under these assumptions, we have derived an exact closed-form expression for the plenoptic spectrum of a slanted plane and then demonstrated that this expression can be used to obtain the plenoptic spectrum of a smoothly varying scene. This is achieved by approximating the surface of the scene with slanted planes. The closed-form expression is then used to determine uniform plenoptic sampling results for a slanted plane. In particular, we derived a new reconstruction filter and a new expression for maximum camera spacing. Using synthetic and real scenes, we showed that the proposed reconstruction filter outperforms previously proposed filters when reconstructing the plenoptic function. Taken as a whole, the analysis of the plenoptic function presented in this paper offer a theoretical basis from which new IBR algorithms can be designed.

\section{APPENDIX A}

In this appendix, we derive the plenoptic spectrum for a slanted plane with complex exponential texture. Starting from (19), we assume a complex exponential texture signal, $g(s)=$ $e^{j \omega_{s} s}$, and obtain

$$
\begin{aligned}
P_{S}\left(\omega_{t}, \omega_{v}\right)= & M_{1} \int_{0}^{T}\left[\operatorname{sinc}\left(\hat{\omega}_{I}\right)-j \bar{v}_{m} \tan (\phi) \operatorname{sinc}^{\prime}\left(\hat{\omega}_{I}\right)\right] \\
& \cdot e^{-j\left(\omega_{t} \cos (\phi)-\omega_{s}\right) s} \cos (\phi) d s .
\end{aligned}
$$

\footnotetext{
${ }^{8}$ Interpolating along the EPI lines assuming a constant gradient of $1 / \eta_{\mathrm{G}}$ is equivalent to rendering assuming a depth of $z_{\mathrm{G}}$.
}

From here we change the variable of integration from $s$ to $\hat{\omega}_{I}$, thus

$s=\frac{\omega_{v} f-z_{\min } \omega_{t}}{\sin (\phi) \omega_{t}}-\hat{\omega}_{I} \frac{1}{\sin (\phi) \omega_{t} \bar{v}_{m}}, \quad d s=d \hat{\omega}_{I} \frac{-1}{\sin (\phi) \omega_{t} \bar{v}_{m}}$, and the limits of integration become $\hat{\omega}_{I}=\omega_{v} v_{m}-\omega_{t} z_{\min } \bar{v}_{m}=$ $b$ when $s=0$ and $\hat{\omega}_{I}=\omega_{v} v_{m}-\omega_{t} z_{\max } \bar{v}_{m}=a$ when $s=T$. Note that the change of variable is only valid for $\omega_{t} \neq 0$; the case when $\omega_{t}=0$ is addressed below. To aid in the following manipulations, we define a new quantity

$$
c=\frac{-\omega_{t} \cos (\phi)+\omega_{s}}{\sin (\phi) \omega_{t} \bar{v}_{m}} .
$$

As a result, (39) becomes

$$
\begin{aligned}
& P_{S}\left(\omega_{t}, \omega_{v}\right)=\frac{j M_{1}}{\omega_{t}} e^{j b c} \int_{b}^{a} \operatorname{sinc}^{\prime}\left(\hat{\omega}_{I}\right) e^{-j c \hat{\omega}_{I}} d \hat{\omega}_{I} \\
& \quad-\frac{f M_{1} e^{j b c}}{\tan (\phi) \omega_{t} v_{m}} \int_{b}^{a} \operatorname{sinc}\left(\hat{\omega}_{I}\right) e^{-j c \hat{\omega}_{I}} d \hat{\omega}_{I} \\
& \stackrel{(i)}{=} \frac{j M_{1} e^{j b c}}{\omega_{t}}\left[\operatorname{sinc}(a) e^{-j a c}-\operatorname{sinc}(b) e^{-j b c}\right] \\
& \quad-\left(\frac{M_{1} \omega_{s} f}{v_{m} \sin (\phi) \omega_{t}^{2}}\right) e^{j b c} \int_{b}^{a} \operatorname{sinc}\left(\hat{\omega}_{I}\right) e^{-j c \hat{\omega}_{I}} d \hat{\omega}_{I},
\end{aligned}
$$

where $(i)$ follows from integration by parts. The final step of the derivation is to rearrange the integral in (40) into four separate integrals as follows

$$
\begin{aligned}
& \int_{b}^{a} \operatorname{sinc}\left(\hat{\omega}_{I}\right) e^{-j c \hat{\omega}_{I}} d \hat{\omega}_{I} \\
& =\frac{1}{2 j}\left(\int_{b}^{a} \frac{1-e^{-j(c+1) \hat{\omega}_{I}}}{\hat{\omega}_{I}} d \hat{\omega}_{I}-\int_{b}^{a} \frac{1-e^{-j(c-1) \hat{\omega}_{I}}}{\hat{\omega}_{I}} d \hat{\omega}_{I}\right) \\
& =\frac{1}{2 j}\left(\int_{0}^{j a(c+1)} \frac{1-e^{-\hat{\omega}_{I}}}{\hat{\omega}_{I}} d \hat{\omega}_{I}-\int_{0}^{j b(c+1)} \frac{1-e^{-\hat{\omega}_{I}}}{\hat{\omega}_{I}} d \hat{\omega}_{I}\right. \\
& \left.\quad-\int_{0}^{j a(c-1)} \frac{1-e^{-\hat{\omega}_{I}}}{\hat{\omega}_{I}} d \hat{\omega}_{I}+\int_{0}^{j b(c-1)} \frac{1-e^{-\hat{\omega}_{I}}}{\hat{\omega}_{I}} d \hat{\omega}_{I}\right) \\
& =\frac{1}{2 j}(\zeta\{j a(c+1)\}-\zeta\{j b(c+1)\} \\
& \quad-\zeta\{j a(c-1)\}+\zeta\{j b(c-1)\}) .
\end{aligned}
$$

Substituting (41) into (40), the expression for the plenoptic spectrum of a slanted plane with complex exponential texture, assuming $\omega_{t} \neq 0$, is

$$
\begin{gathered}
P_{S}\left(\omega_{t}, \omega_{v}\right)=\left(\frac{j 2 v_{m}}{\omega_{t}}\left[\operatorname{sinc}(a) e^{-j T\left(\omega_{t} \cos (\phi)-\omega_{s}\right)}-\operatorname{sinc}(b)\right]\right. \\
+\frac{j \omega_{s} f}{\sin (\phi) \omega_{t}^{2}}[\zeta\{j b(c-1)\}-\zeta\{j a(c-1)\}-\zeta\{j b(c+1)\} \\
\left.+\zeta\{j a(c+1)\}] e^{j b c}\right) e^{-j \omega_{t} x_{1}} .
\end{gathered}
$$

If $\omega_{t}=0$, the integral in (39) may be evaluated directly to obtain

$$
\begin{aligned}
P_{S}\left(0, \omega_{v}\right)= & 2 v_{m} T \operatorname{sinc}\left(\frac{\omega_{s} T}{2}\right)\left[\cos (\phi) \operatorname{sinc}\left(\omega_{v} v_{m}\right)\right. \\
& \left.-j \frac{\sin (\phi) v_{m}}{f} \operatorname{sinc}^{\prime}\left(\omega_{v} v_{m}\right)\right] e^{j \omega_{s} \frac{T}{2}} .
\end{aligned}
$$


The function $\zeta$ is defined in (22).

\section{APPENDIX B}

In this appendix we derive the plenoptic spectrum for a slanted plane with bandlimited texture. Starting from the equation for the plenoptic spectrum defined in (19), we represent both the texture signal, $g(s)$, and the finite limits of the integral with the function

$$
h(s)=\operatorname{rect}\left(\frac{s}{T}-\frac{1}{2}\right) g(s)= \begin{cases}g(s), & \text { if } 0 \leq s \leq T \\ 0, & \text { else. }\end{cases}
$$

Therefore we obtain the following equation for the plenoptic spectrum

$$
\begin{gathered}
P_{S}\left(\omega_{t}, \omega_{v}\right)=M_{1} \cos (\phi)\left(\int_{-\infty}^{\infty} h(s) \operatorname{sinc}\left(\hat{\omega}_{I}\right) e^{-j \omega_{t} \cos (\phi) s} d s\right. \\
\left.-j \bar{v}_{m} \tan (\phi) \int_{-\infty}^{\infty} h(s) \operatorname{sinc}^{\prime}\left(\hat{\omega}_{I}\right) e^{-j \omega_{t} \cos (\phi) s} d s\right), \text { (44) }
\end{gathered}
$$

remembering that $\hat{\omega}_{I}=\omega_{v} v_{m}-\left(s \sin (\phi)+z_{\min }\right) \bar{v}_{m} \omega_{t}$. At this point, we define $H(\Omega)$ as the Fourier transform of $h(s)$ and $\Omega$ as its the frequency variable. Consequently the inverse Fourier transform is

$$
h(s)=\mathcal{F}_{\Omega}^{-1}\{H(\Omega)\}=\frac{1}{2 \pi} \int_{-\infty}^{\infty} H(\Omega) e^{j \Omega s} d \Omega .
$$

If we now substitute this inverse transform into (44) and change the order of integration, the plenoptic spectrum becomes

$$
\begin{gathered}
P_{S}\left(\omega_{t}, \omega_{v}\right)=\frac{M_{1}}{2 \pi} \cos (\phi)\left(\int_{-\infty}^{\infty} H(\Omega) Q_{1}\left(\omega_{t}, \omega_{v}, \Omega\right) d \Omega\right. \\
\left.-j \bar{v}_{m} \tan (\phi) \int_{-\infty}^{\infty} H(\Omega) Q_{2}\left(\omega_{t}, \omega_{v}, \Omega\right) d \Omega\right),
\end{gathered}
$$

where

$$
Q_{1}\left(\omega_{t}, \omega_{v}, \Omega\right)=\int_{-\infty}^{\infty} \operatorname{sinc}\left(\hat{\omega}_{I}\right) e^{-j \omega_{t} \cos (\phi) s} e^{j \Omega s} d s
$$

and

$$
Q_{2}\left(\omega_{t}, \omega_{v}, \Omega\right)=\int_{-\infty}^{\infty} \operatorname{sinc}^{\prime}\left(\hat{\omega}_{I}\right) e^{-j \omega_{t} \cos (\phi) s} e^{j \Omega s} d s .
$$

The integrals $Q_{1}\left(\omega_{t}, \omega_{v}, \Omega\right)$ and $Q_{2}\left(\omega_{t}, \omega_{v}, \Omega\right)$ are solved by rearranging them into Fourier transforms in which $\Omega$ is the frequency variable.

Starting with $Q_{1}\left(\omega_{t}, \omega_{v}, \Omega\right)$, we use the definition of $\hat{\omega}_{I}$ and the following Fourier transform identities

$$
\mathcal{F}_{s}\left\{\operatorname{sinc}(\alpha(s-\beta)\}=\frac{\pi}{\alpha} \operatorname{rect}\left(\frac{\Omega}{2 \alpha}\right) e^{-j \Omega \beta},\right.
$$

and $\mathcal{F}_{s}\left\{e^{j \alpha s}\right\}=\delta(\Omega-\alpha)$, where $\alpha, \beta \in \mathbb{R}$ and $\delta$ is the delta Dirac, to obtain

$$
\begin{aligned}
Q_{1}\left(\omega_{t}, \omega_{v}, \Omega\right)= & \frac{\pi}{\sin (\phi) \bar{v}_{m} \omega_{t}} \operatorname{rect}\left(\frac{\Omega-\omega_{t} \cos (\phi)}{2 \sin (\phi) \bar{v}_{m} \omega_{t}}\right) \\
& \cdot e^{-j\left(\frac{z_{\min }}{\sin (\phi)}-\frac{\omega_{v} f}{\sin (\phi) \omega_{t}}\right)\left(\Omega-\omega_{t} \cos (\phi)\right)} .
\end{aligned}
$$

Likewise, using the transform identities highlighted above and differential property of the Fourier transform, $Q_{2}\left(\omega_{t}, \omega_{v}, \Omega\right)$ becomes

$$
\begin{aligned}
& Q_{2}\left(\omega_{t}, \omega_{v}, \Omega\right)=j \pi\left(\frac{1}{\sin (\phi) \bar{v}_{m} \omega_{t}}\right)^{2}\left(\Omega-\omega_{t} \cos (\phi)\right) \\
& \cdot \operatorname{rect}\left(\frac{\Omega-\omega_{t} \cos (\phi)}{2 \sin (\phi) \bar{v}_{m} \omega_{t}}\right) e^{-j\left(\frac{z_{\min }}{\sin (\phi)}-\frac{\omega_{v} f}{\sin (\phi) \omega_{t}}\right)\left(\Omega-\omega_{t} \cos (\phi)\right)} .
\end{aligned}
$$

Finally, if we substitute (49) and (50) into (46) and rearrange, we obtain the plenoptic spectrum for a slanted plane with bandlimited texture:

$$
\begin{gathered}
P_{S}\left(\omega_{t}, \omega_{v}\right)=\int_{-\infty}^{\infty} H(\Omega) \frac{f \Omega}{\sin (\phi) \omega_{t}^{2}} \operatorname{rect}\left(\frac{\Omega-\omega_{t} \cos (\phi)}{2 \sin (\phi) \bar{v}_{m} \omega_{t}}\right) \\
\cdot e^{-j \Omega\left(\frac{z_{\min }}{\sin (\phi)}-\frac{\omega_{v f}}{\sin (\phi) \omega_{t}}\right)} e^{-j \omega_{t}\left(x_{1}-\frac{z_{\min }}{\tan (\phi)}\right)} e^{-j \omega_{v}\left(\frac{f}{\tan (\phi)}\right)} d \Omega .
\end{gathered}
$$

\section{APPENDIX C}

We start by setting $\omega_{v}=\omega_{t} z_{\max } / f$ in (24), hence we obtain the following 1D slice of the plenoptic spectrum

$$
\begin{gathered}
P_{S}\left(\omega_{t}, \omega_{t} \frac{z_{\max }}{f}\right)=e^{-j \omega_{t} x_{2}} \int_{-\infty}^{\infty} H(\Omega) \frac{f \Omega}{\sin (\phi) \omega_{t}^{2}} \\
\quad \cdot \operatorname{rect}\left(\frac{\Omega-\omega_{t} \cos (\phi)}{2 \sin (\phi) \bar{v}_{m} \omega_{t}}\right) e^{-j \Omega T} d \Omega .
\end{gathered}
$$

To determine the essential bandwidth of this spectrum we examine the spectral behaviour of the integrand.

From (23), it follows that $H(\Omega)=G(\Omega) * W(\Omega)$ where $G(\Omega)$ is the Fourier transform of $g(s), *$ denotes convolution and

$$
W(\Omega)=\mathcal{F}\left\{\operatorname{rect}\left(\frac{s}{T}-\frac{1}{2}\right)\right\}=T \operatorname{sinc}\left(\frac{\Omega T}{2}\right) e^{-j \frac{\Omega}{2} T} .
$$

Consequently the essential bandwidth of $H(\Omega)$ is the sum of the essential bandwidth of $W(\Omega)$ and the bandwidth of $G(\Omega)$. Using [28], the essential bandwidth of $W(\Omega)$ is

$$
B_{W}=\left\{\Omega:|\Omega| \leq \frac{2 \pi}{T}\right\}
$$

which means the essential bandwidth of $H(\Omega)$ is

$$
B_{H}=\left\{\Omega:|\Omega| \leq \omega_{s}+\frac{2 \pi}{T}\right\},
$$

since $G(\Omega)$ is bandlimited to $\omega_{s}$.

Next, from the definition of the rect function, the integrand in (52) is non-zero when the following inequality is satisfied

$$
-\frac{1}{2} \leq \frac{\Omega f-\omega_{t} \cos (\phi) f}{2 \sin (\phi) v_{m} \omega_{t}} \leq \frac{1}{2} .
$$

Therefore, if we assume $H(\Omega)$ is approximately bandlimited to (55), the above inequality can be rearranged to give a region in $\omega_{t}$ for which (52) is non-zero. Using this region, we obtain the essential bandwidth of (52) as

$$
B_{t}=\left\{\omega_{t}:\left|\omega_{t}\right| \leq \frac{1}{\cos (\phi)-\bar{v}_{m} \sin (\phi)\left(\omega_{s}+\frac{2 \pi}{T}\right)}\right\} \text {. }
$$




\section{APPENDIX D}

Using (27), we set $\omega_{t}=\Omega_{t}$ and substitute the definition into (24) to obtain

$$
\begin{gathered}
P_{S}\left(\Omega_{t}, \omega_{v}\right)=\frac{f^{2}}{\sin (\phi) \Omega_{t}^{2}} \int_{-\infty}^{\infty} H(\Omega) \operatorname{rect}\left(\frac{\Omega f-f \Omega_{t} \cos (\phi)}{2 v_{m} \Omega_{t} \sin (\phi)}\right) \\
\cdot \Omega e^{j \Omega\left(\frac{\omega_{v} f-z_{\min } \Omega_{t}}{\Omega_{t} \sin (\phi)}\right)} d \Omega e^{j\left(\frac{z_{\min }}{\tan (\phi)}-x_{1}\right) \Omega_{t}-j \omega_{v} \frac{f}{\tan (\phi)},}
\end{gathered}
$$

Using Fourier transform theory, the integral in (58) can be expressed as follows

$$
\mathcal{F}_{\Omega}^{-1}\{H(\Omega)\} * \mathcal{F}_{\Omega}^{-1}\left\{\Omega \operatorname{rect}\left(\frac{\Omega f-f \Omega_{t} \cos (\phi)}{2 v_{m} \Omega_{t} \sin (\phi)}\right)\right\},
$$

where $*$ denotes convolution. As a result, the overall essential bandwidth, $B_{v}$, is the sum of the individual bandwidths corresponding to each inverse Fourier transform in (59).

Starting with the first transform, from the identity in (43), it follows that

$$
\begin{aligned}
\mathcal{F}_{\Omega}^{-1}\{H(\Omega)\}= & 2 \pi g\left(\frac{\omega_{v} f-z_{\min } \Omega_{t}}{\sin (\phi) \Omega_{t}}\right) \\
& \cdot \operatorname{rect}\left(\frac{\omega_{v} f-z_{\min } \Omega_{t}}{T \sin (\phi) \Omega_{t}}-\frac{1}{2}\right) .
\end{aligned}
$$

Therefore, using the definition of the rect function, the above expression is non-zero only when

$$
\frac{z_{\min }}{f} \Omega_{t} \leq \omega_{v} \leq \frac{z_{\max }}{f} \Omega_{t},
$$

thus we have the bandwidth for the first inverse Fourier transform in (59).

Next, using the derivative identity of the Fourier transform, we obtain the following expression for the second inverse Fourier transform in (59):

$$
\begin{aligned}
\mathcal{F}_{\Omega}^{-1} & \left\{\Omega \operatorname{rect}\left(\frac{\Omega f-f \Omega_{t} \cos (\phi)}{2 v_{m} \Omega_{t} \sin (\phi)}\right)\right\}=M_{2} e^{j \cot (\phi)\left(\omega_{v} f-z_{\min } \Omega_{t}\right)} \\
\cdot & {\left[f \cos (\phi) \operatorname{sinc}\left(\omega_{v} v_{m}-z_{\min } \frac{\Omega_{t} v_{m}}{f}\right)\right.} \\
& \left.-j v_{m} \sin (\phi) \operatorname{sinc}^{\prime}\left(\omega_{v} v_{m}-z_{\min } \frac{\Omega_{t} v_{m}}{f}\right)\right]
\end{aligned}
$$

where

$$
M_{2}=2 v_{m} \sin (\phi)\left(\frac{\Omega_{t}}{f}\right)^{2} \text {. }
$$

The essential bandwidth of this expression depends on the spectral behaviour of the sinc function and its derivative. From [28], we know that the essential bandwidth of the sinc function in $(62)$ is

$$
B_{1}=\left\{\omega_{v}: \omega_{v} \in\left[\Omega_{t} \frac{z_{\min }}{f}-\frac{\pi}{v_{m}}, \Omega_{t} \frac{z_{\min }}{f}+\frac{\pi}{v_{m}}\right]\right\},
$$

and, using the same method as [28], the essential bandwidth of the derivative of a sinc function is

$$
B_{2}=\left\{\omega_{v}: \omega_{v} \in\left[\Omega_{t} \frac{z_{\min }}{f}-\frac{3.5 \pi}{v_{m}}, \Omega_{t} \frac{z_{\min }}{f}+\frac{3.5 \pi}{v_{m}}\right]\right\} .
$$

In view of this, we propose that the essential bandwidth for (62) is the weighted combination of $B_{1}$ and $B_{2}$. The weights are based on the proportion of energy contributed by that function to the total energy of (62). Therefore, if $\mathcal{E}_{\text {sinc }}$ and $\mathcal{E}_{\text {dsinc }}$ represent the energy for the sinc and its derivative, respectively, then the essential bandwidth of (62) is

$$
\left(\frac{\mathcal{E}_{\text {sinc }}}{\mathcal{E}_{\text {sinc }}+\mathcal{E}_{\text {dsinc }}}\right) B_{1}+\left(\frac{\mathcal{E}_{\text {dsinc }}}{\mathcal{E}_{\text {sinc }}+\mathcal{E}_{\text {dsinc }}}\right) B_{2} \text {. }
$$

The energies $\mathcal{E}_{\text {sinc }}$ and $\mathcal{E}_{\text {dsinc }}$ are defined as follows:

$$
\mathcal{E}_{\text {sinc }}=M_{2}^{2}(f \cos (\phi))^{2} \frac{\pi}{v_{m}}
$$

and

$$
\mathcal{E}_{\mathrm{dsinc}}=M_{2}^{2}\left(v_{m} \sin (\phi)\right)^{2} \frac{\pi}{3 v_{m}} .
$$

Therefore, the complete essential bandwidth for (62) is

$$
\begin{gathered}
B_{3}=\left\{\omega_{v}: \omega_{v} \in\left[\Omega_{t} \frac{z_{\min }}{f}-n(\phi, \bar{v}) \frac{\pi}{v_{m}},\right.\right. \\
\left.\left.\Omega_{t} \frac{z_{\min }}{f}+n(\phi, \bar{v}) \frac{\pi}{v_{m}}\right]\right\},
\end{gathered}
$$

where

$$
n\left(\phi, \bar{v}_{m}\right)=\frac{3 \cos ^{2}(\phi)+3.5\left(\bar{v}_{m} \sin (\phi)\right)^{2}}{3 \cos ^{2}(\phi)+\left(\bar{v}_{m} \sin (\phi)\right)^{2}} .
$$

As a result, using (61) and (66), the essential bandwidth for (58) is

$$
\begin{gathered}
B_{v}=\left\{\omega_{v}: \omega_{v} \in\left[\Omega_{t} \frac{z_{\min }}{f}-n(\phi, \bar{v}) \frac{\pi}{v_{m}},\right.\right. \\
\left.\left.\Omega_{t} \frac{z_{\max }}{f}+n(\phi, \bar{v}) \frac{\pi}{v_{m}}\right]\right\},
\end{gathered}
$$

\section{REFERENCES}

[1] M. N. Do, Q. H. Nguyen, H. T. Nguyen, D. Kubacki, and S. J. Patel, "Immersive visual communication," IEEE Signal Process. Mag., vol. 28, no. 1, pp. 58-66, 2011.

[2] A. Kubota, A. Smolic, M. Magnor, M. Tanimoto, T. Chen, and C. Zhang, "Multiview imaging and 3DTV," IEEE Signal Process. Mag., vol. 24, no. 6, pp. 10-21, 2007.

[3] M. Tanimoto, M. P. Tehrani, T. Fujii, and T. Yendo, "Free-viewpoint TV," IEEE Signal Process.Mag., vol. 28, no. 1, pp. 67-76, 2011.

[4] C. Zhang and T. Chen, "A survey on image-based rendering - representation, sampling and compression," EURASIP Signal Processing: Image Communication, vol. 19, no. 1, pp. 1-28, 2004.

[5] H. Y. Shum, S. C. Chan, and S. B. Kang, Image-Based Rendering. Springer, 2007.

[6] E. H. Adelson and J. R. Bergen, "The plenoptic function and the elements of early vision," in Computational Models of Visual Processing. MIT Press, Cambridge, MA, 1991, pp. 3-20.

[7] J.-X. Chai, X. Tong, S.-C. Chan, and H.-Y. Shum, "Plenoptic sampling," in Proc. SIGGRAPH, New York, NY, 2000, pp. 307-318.

[8] C. Zhang and T. Chen, "Spectral analysis for sampling image-based rendering data," IEEE Trans. Circuits and Syst. Video Technol., vol. 13, no. 11 , pp. 1038-1050, 2003.

[9] M. N. Do, D. Marchand-Maillet, and M. Vetterli, "On the bandwidth of the plenoptic function," IEEE Trans. Image Process., vol. 21, no. 2, pp. 708-717, 2012.

[10] C. Chen and D. Schonfeld, "Geometrical plenoptic sampling," in Proc. Int. Conf. Image Process. (ICIP), Cairo, Egypt, 2009, pp. 3769-3772.

[11] L. Bagnato, P. Frossard, and P. Vandergheynst, "Plenoptic spherical sampling," in Proc. Int. Conf. Image Process. (ICIP), Florida, FL, 2012.

[12] C. Zhang and T. Chen, Light Field Sampling, ser. Synthesis Lectures on Image, Video, and Multimedia Processing. Morgan \& Claypool Publishers, 2006.

[13] C. Gilliam, P. L. Dragotti, and M. Brookes, "A closed-form expression for the bandwidth of the plenoptic function under finite field of view constraints," in Proc. IEEE Int. Conf. Image Process. (ICIP), Hong Kong, 2010, pp. 3965-3968. 
[14] J. Shade, S. Gortler, L. W. He, and R. Szeliski, "Layered depth images," in Proc. SIGGRAPH, Orlando, FL, 1998, pp. 231-242.

[15] H. Y. Shum, J. Sun, S. Yamazaki, Y. Li, and C. K. Tang, "Pop-up light field: An interactive image-based modeling and rendering system," $A C M$ Trans. Graphics, vol. 23, pp. 143-162, 2004.

[16] J. Berent and P. L. Dragotti, "Plenoptic manifolds," IEEE Signal Process. Mag., vol. 24, no. 6, pp. 34-44, 2007.

[17] J. Pearson, M. Brookes, and P. L. Dragotti, "Plenoptic layer-based modeling for image based rendering," IEEE Trans. Image Process., vol. 22, no. 9, pp. 3405-3419, 2013.

[18] L. McMillan and G. Bishop, "Plenoptic modelling: An image-based rendering system," in Proc. SIGGRAPH, Los Angeles, CA, 1995, pp. $39-46$.

[19] H. Y. Shum, S. B. Kang, and S. C. Chan, "Survey of image-based representations and compression techniques," IEEE Trans. Circuits Syst. Video Technol., vol. 13, no. 11, pp. 1020-1037, 2003.

[20] M. Levoy and P. Hanrahan, "Light field rendering," in Proc. SIGGRAPH, New Orleans, LA, 1996, pp. 31-40.

[21] S. Gortler, R. Grzeszczuk, R. Szeliski, and M. Cohen, "The lumigraph," in Proc. SIGGRAPH, New York, NY, 1996, pp. 43-54.

[22] T. Fujii, T. Kimoto, and M. Tanimoto, "Ray space coding for 3D visual communication," in Proc. Picture Coding Symp., 1996, pp. 447?-451.

[23] G. Miller, S. Rubin, and D. Ponceleon, "Lazy decompression of surface light fields for precomputed global illumination," in Proc. Rendering Techniques, 1998, pp. 281-292.

[24] D. Wood, D. Azuma, K. Aldinger, B. Curless, T. Duchamp, D. Salesin, and W. Stuetzle, "Surface light fields for 3D photography," in Proc. SIGGRAPH, New Orleans, LA, 2000, pp. 287-296.

[25] H. T. Nguyen and M. N. Do, "Error analysis for image-based rendering with depth information," IEEE Trans. Image Process., vol. 18, no. 4, pp. 703-716, 2009.

[26] R. Bolles, H. Baker, and D. Marimont, "Epipolar-plane image analysis: An approach to determining structure from motion," Int. J. Computer Vision, vol. 1, no. 1, pp. 7-55, 1987.

[27] M. Abramowitz and I. A. Stegun, Eds., Handbook of Mathematical Functions with Formulas, Graphs, and Mathematical Tables. Dover, 1964.

[28] B. P. Lathi, Modern Digital and Analog Communication Systems. Oxford University Press, 1998.

[29] T. Blu, P. Thévenaz, and M. Unser, "MOMS: Maximal-order interpolation of minimal support," IEEE Trans. Image Process., vol. 10, no. 7, pp. $1069-1080,2001$.

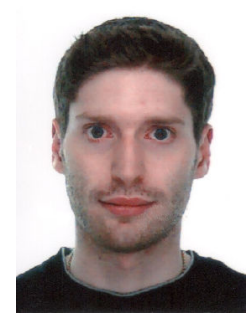

Christopher Gilliam is a Postdoctoral Fellow in the Image and Video Processing Group, Department of Electronic Engineering at The Chinese University of Hong Kong. He graduated from Imperial College London with a First Class Honours MEng in Electrical and Electronic Engineering in 2008 and with a $\mathrm{PhD}$ in Electrical and Electronic Engineering in 2013. His research interests include sampling theory, approximation theory and its connection to the sampling of parametric non-bandlimited signals, and applications of sampling theory to image processing, in particular Image-Based Rendering (IBR).

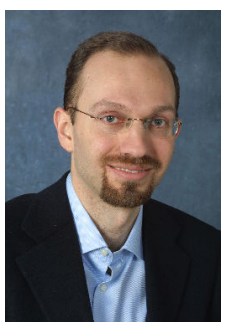

Pier Luigi Dragotti is currently a Reader (Associate Professor) with the Electrical and Electronic Engineering Department at Imperial College London, U.K. He received the Laurea degree (summa cum laude) in electrical and electronic engineering from the University of Naples Federico II, Naples, Italy, in 1997; the masters degree in communications systems from the Swiss Federal Institute of Technology of Lausanne (EPFL), Lausanne, Switzerland, in 1998, and the Ph.D. degree from EPFL in April 2002. $\mathrm{He}$ has held several visiting positions at different universities and research centers including: Visiting Student with Stanford University, CA, USA, in 1996, Visiting Researcher with the Mathematics of Communications Department, Bell Labs, Lucent Technologies, Murray Hill, NJ, USA, in 2000, Visiting Professor with the Politecnico of Milan, Italy, in 2010, and Visiting Scientist with the Massachusetts Institute of Technology (MIT), Cambridge, MA, USA, in 2011. He was Technical Co-Chair for the European Signal Processing Conference in 2012, a Associate Editor of the IEEE TRANSACTIONS ON IMAGE PROCESSING from 2006 to 2009 and an Elected Member of the IEEE Image, Video and Multidimensional Signal Processing Technical Committee and the IEEE Signal Processing Theory and Methods Technical Committee. He is a recipient of the ERC Starting Investigator Award for the project RecoSamp. His research interests include sampling theory, wavelet theory and its applications, image and video compression, image-based rendering, and image super-resolution.

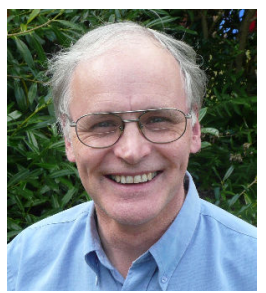

Mike Brookes is a Reader (Associate Professor) in the Communications and Signal Processing Research Group of the Department of Electrical and Electronic Engineering at Imperial College London. He obtained his first degree in Mathematics from Cambridge University in 1972 and then spent four years at the Massachusetts Institute of Technology (1973-77) before returning to the UK and joining Imperial College. His research focuses on speech processing and computer vision. Within the area of speech processing, he has concentrated on the modelling and analysis of speech signals, the extraction of features for speech and speaker recognition and, most recently, on the enhancement of poor quality speech signals. Between 2007 and 2012, he was the Director of the Home Office sponsored Centre for Law Enforcement Audio Research (CLEAR) which investigated techniques for processing heavily corrupted speech signals. In image processing he led a successful MoD-funded project on radar target recognition in partnership with General Dynamics and is currently working on a number of computer vision projects concerned with change detection and image-based rendering from multiperspective camera systems. 